\title{
Neutral sphingomyelinase 1 regulates cellular fitness at the level of ER stress and cell cycle
}

Dolma Choezom¹, and Julia Christina Gross ${ }^{1,2,3}$

\section{Affiliations:}

1 Developmental Biochemistry, University Medical Center Goettingen, Goettingen, Germany

2 Hematology and Oncology, University Medical Center Goettingen, Goettingen, Germany

${ }^{3}$ Health and Medical University, Potsdam, Germany

\section{*Correspondence:}

Julia Christina Gross, Health and Medical University, Potsdam, Germany

\section{julia.gross@health-and-medical-university.de}

\section{Abstract}

Neutral sphingomyelinase 1 (nSMase1) belongs to the sphingomyelinase enzyme family that hydrolyzes sphingomyelin to produce signaling active lipid ceramide and phosphorylcholine. The molecular characterization and biological function of nSMase1 remain poorly studied. Here, we report that nSMase1 (gene name: SMPD2) knockdown reduces LAMP1 at the mRNA levels and is required for initiating a full-potential unfolded protein response under ER stress. Additionally, SMPD2 KD dramatically reduces the global protein translation rate. We further show that SMPD2 KD cells are arrested in the G1 phase of the cell cycle and that two important cell cycle regulating processes PI3K/Akt pathway and Wnt signaling pathway are altered. Taken together, we propose a role for nSMase1 in buffering ER stress and modulating cellular fitness via cell cycle regulation.

\section{Keywords}

Cell cycle arrest, Wnt-signaling, Ceramide, PI3K/AKT, ER stress, LAMP1, translation 


\section{Introduction}

Sphingomyelin, a major lipid constituent of the plasma membrane, through its catabolic metabolism generates several bioactive signaling lipids: ceramide, sphingosine, and sphingosine1-phosphate (Hannun \& Obeid, 2008). Sphingomyelindiesterases (SMPDs) or sphingomyelinases (SMases) are a family of enzymes that hydrolyze the phosphodiester bond of the sphingomyelin to produce ceramide and phosphorylcholine (Airola \& Hannun, 2013). The sphingomyelinases are broadly divided into three major classes: acid sphingomyelinases, alkaline sphingomyelinases, and neutral sphingomyelinases based on their pH optima (Airola \& Hannun, 2013). In mammals, four neutral sphingomyelinase members have been identified, nSMase1 (Gene name: SMPD2) ((Tomiuk et al, 1998)), nSMase2 (SMPD3), nSMase3 (SMPD4), and mitochondrial associated nSMase (MA-nSMase; SMPD5) (Yabu et al, 2009; Wu et al, 2010).

We have previously shown that nSMase 2 activity at the endosomal membrane regulates exosome secretion by counteracting V-ATPase-mediated endosomal acidification (Choezom \& Gross, 2022). The number and different cellular localization of the neutral sphingomyelinase family members suggest distinct functions for each enzyme. NSMase1 shares identical domain architecture to ISC1, the yeast homolog to neutral sphingomyelinases (Tomiuk et al, 1998; Hofmann et al, 2000). In contrast to the overexpressed nSMase1 which localizes to the endoplasmic reticulum (Fensome et al, 2000; Rodrigues-Lima et al, 2000; Hofmann et al, 2000), endogenous nSMase1 exclusively localizes to the nuclear matrix (Mizutani et al, 2001). Although nSMase1 hydrolyzes sphingomyelin in-vitro, sphingomyelin metabolism remains unaffected upon its overexpression in cells (Tomiuk et al, 1998; Sawai et al, 1999). Likewise, nSMase1 mouse knockout showed no obvious phenotype with no detectable changes in sphingomyelin metabolism (Zumbansen \& Stoffel, 2002). Instead of sphingomyelin, a study has shown that nSMase1 hydrolyzes lyso-platelet-activating factor (lyso-PAF) invitro and in cells (Sawai et al, 1999). This indicates the putative nSMase1 could be a lysoPAF phospholipase C with lyso-PAF as its biological substrate (Sawai et al, 1999). Despite being the first neutral sphingomyelinase in mammals to get cloned and identified, the biochemical and molecular characterization of nSMase 1 remains poorly studied. 
In contrast to the above biochemical studies, several studies showed that ceramide generated by nSMase1 activity induces cellular death via different pathways under different stress conditions (Tonnetti et al, 1999; Jaffrézou et al, 1996; Jana \& Pahan, 2004; Lee et al, 2004). JNK-signaling activates nSMase1 by phosphorylation to generate ceramide for apoptosis induction upon different environmental stresses such as heat shock and UV irradiation (Yabu et al, 2015). Given the insufficient and contrasting results on the role of nSMase1, we aimed to ascertain the biological role of nSMase1 by using siRNA-mediated gene knockdown (KD) in two human cell lines: HCT116, a human colorectal cancer cell line, and HeLa, a cervical cancer line.

Here, we discover that nSMase1 plays an important role in maintaining overall cellular homeostasis. SMPD2 KD reduces LAMP1 (lysosomal-associated membrane protein 1) proteins levels by downregulating its mRNA. And this dramatic reduction in LAMP1 protein has no apparent effect on the function of lysosomes as autophagy remained unaffected. Interestingly, SMPD2 KD cells are not only inefficient in activating a fullpotential unfolded protein response (UPR) signaling upon ER stress but are arrested in the G1 phase of the cell cycle. We further show that two important cell cycle regulating processes - PI3K/Akt pathway and Wnt signaling pathway- are altered by SMPD2 KD. Specifically, SMPD2 KD affects PI3K/Akt signaling by significantly reducing the level of phosphorylated Akt. Most importantly, SMPD2 KD significantly reduces the Wnt signaling activity by reducing $\beta$-catenin protein levels in HeLa cells and Wnt3a in HCT116 at both protein and mRNA levels. Additionally, SMPD2 dramatically reduces the overall protein translation rate. These findings prove an important biological role for nSMase1 and provide strong foundations for further studies on dissecting its molecular function and pathway.

\section{Results}

\section{SMPD2 Knockdown downregulates LAMP1 mRNA level}

Sphinghomylinases are active at different cellular membranes (Airola \& Hannun, 2013). We have previously shown that nSMase2 (gene name: SMPD3) activity at the endosomal membrane regulates exosome secretion by counteracting V-ATPase-mediated endosomal acidification (Choezom \& Gross, 2022). Here, we analyzed the potential role 
of nSMase1 (gene name: SMPD2), a less well-understood member of the neutral sphingomyelinase family, in the regulation of endosomal trafficking decisions. SMPD2 knockdown (KD) significantly reduced HeLa cell viability when compared with control cells. In comparison, KD of SMPD3, NSMAF, an activating factor of nSMase2 (Philipp et al, 2010), and Syntenin (gene name: SDCBP), an adaptor protein involved in exosome biogenesis (Baietti et al, 2012), did not affect cell viability(Fig. S1A). These data indicate that SMPD2 is a vital gene for cellular survival. Upon SMPD3 KD, endosomal markers and acidified compartments accumulate intracellularly (Choezom \& Gross, 2022). SMPD3 KD also slightly increased lysosomal protein LAMP1 levels, in contrast, SMPD2 KD strongly downregulated LAMP1 in both HeLa (Fig. 1A, Fig. S1B) and HCT116 (Fig. S1C). NSMAF and Syntenin (gene name: SDCBP) KD did not alter LAMP1 protein levels (Fig. 1A). Interestingly, SMPD2 KD did not affect lysosomal acidification as the total Lysotracker puncta per cell remained unaffected by SMPD2 KD (Fig. 1B, C). To directly determine whether the downregulation of LAMP1 protein upon SMPD2 KD affects lysosomal activity, we analyzed autophagy flux with two known markers - LC3B-II and P62 - by measuring their lysosomal turnover under both serum-fed and serum-starvation conditions with and without lysosomal inhibition with Bafilomycin-A (BAF) (Tanida et al, 2005). We found that P62 and LC3B-II flux remained unchanged upon SMPD2 KD under both basal and serum starvation conditions (Fig. 1D-F). These data allow two conclusions: Firstly, upon SMPD2 KD, downregulated LAMP1 does not affect lysosomal function. Secondly, as LAMP1 protein levels remained downregulated even upon lysosomal inhibition (Fig. 1D), we concluded that SMPD2 KD does not downregulate LAMP1 proteins through lysosomal degradation.

We next analyzed whether SMPD2 KD downregulates LAMP1 through proteasomal degradation by using proteasomal inhibitor MG132. Efficient proteasomal inhibition was demonstrated by accumulation of $\beta$-catenin and ER-resident protein BiP compared with control cells (Fig. 1G), but MG132 did not affect SMPD2 KD-induced LAMP1 downregulation, indicating that SMPD2 KD does not downregulate LAMP1 by proteasomal degradation (Fig. 1G, Fig. S1D). As LAMP1 protein was neither targeted for lysosomal nor proteasomal degradation in SMPD2 KD cells, we next analyzed LAMP1 mRNA levels by qPCR (Fig. 1H). Indeed, the LAMP1 mRNA level was significantly 
reduced by SMPD2 KD in both HeLa (Fig. 1H) and HCT116 (Fig. S1E) cell lines. In contrast, the mRNA level of LAMP2, another closely related lysosomal membrane protein, remained unchanged upon SMPD2 KD (Fig. S1F). Overall, these data confirm that SMPD2 KD specifically downregulates LAMP1 not via protein degradation pathways but at the mRNA level.

\section{SMPD2 KD causes inefficient activation of the UPR upon ER stress induction}

As a readout for nSMase1 activity, we used a ceramide antibody previously used for sphingomyelin metabolism and ceramide signaling studies (Vielhaber et al, 2001; Parashuraman \& D’Angelo, 2019; Yabu et al, 2015). Confocal microscopy analysis upon SMPD2 KD showed a significant reduction of staining in both mean intensity of the intracellular ceramide puncta and mean intensity of ceramide puncta that colocalize with the ER marker Calnexin (Fig. S1G, H). These data suggest that SMPD2 KD affects cellular ceramide levels specifically at the ER. In addition to the reduced ceramide levels at the ER upon SMPD2 KD (Fig. S1G, H), overexpressed nSMase1 localizes to the ER (Rodrigues-Lima et al, 2000; Fensome et al, 2000; Tomiuk et al, 2000), where transmembrane proteins such as LAMP1 are translated (Schwarz \& Blower, 2016). In line with this, we found nSMase1 protein levels significantly increased upon ER stress induction with tunicamycin (Tuni) or thapsigargin (Thapsig) in HeLa cells (Fig. 2A, B). As nSMase1 protein level was increased by ER stress, we next investigated if SMPD2 KD induces ER stress and therefore activates the unfolded protein response (UPR) pathways. ER morphology appeared unchanged upon SMPD2 KD (Fig. S1I). We analyzed three ER stress markers, BiP, Calnexin, and PDI (Protein disulfide-isomerase) which are usually upregulated through UPR pathways upon ER stress induction (Oslowski \& Urano, 2011) (Fig. 2C-F). We found BiP to be upregulated at the protein level by both inhibitors, which was slightly impaired by SMPD2 KD after $4 \mathrm{~h}$ of treatment (Fig. 2C, D). We next analyzed different markers that are upregulated through UPR activation upon ER stress at the mRNA level. Although SMPD2 KD alone did not activate UPR, SMPD2 KD cells failed to upregulate several ER stress markers (sXBP-1, ATF4, and EDEM) to the same levels as their control counterparts upon stress induction (Fig. 2GK). Furthermore, under ER stress conditions the LAMP1 mRNA remained significantly 
downregulated by SMPD2 KD (Fig. 2L). And in alignment with the increased nSMase1 protein levels (Fig. 2A, B), SMPD2 mRNA levels were slightly increased by tunicamycin and thapsigargin treatment (Fig. 2M).

The UPR activation upon ER stress results in mRNA degradation through IRE-1 RNase activity (the RIDD pathway) to relieve stress by reducing protein translation burden in the ER (Hollien et al, 2009). LAMP1 mRNA was identified as a substrate of IRE-1-dependent decay of mRNAs (Maurel et al, 2014). If LAMP1 mRNA is degraded through the RIDD pathway upon SMPD2 KD, we hypothesized that IRE-1 RNase activity inhibitor $4 \mu 8 \mathrm{C}$ (Cross et al, 2012) should restore LAMP1 mRNA and protein levels. IRE-1 cleaves XBP1 (X-box binding protein) mRNA into two transcripts, known as spliced XBP1 and 2 (sXPB1 and 2) (Maurel et al, 2014). The efficient inhibition of the IRE-1 RNase activity by $4 \mu 8 \mathrm{C}$ was confirmed by the absence of spliced XBP-1 (sXBP-1) mRNA (Fig. S1J). However, LAMP1 mRNA, as well as protein levels, remained downregulated, when cells were treated with $4 \mu 8 \mathrm{C}$ overnight after $48 \mathrm{~h}$ of SMPD2 KD (Fig. S1K, L). Surprisingly, even under ER stress conditions induced by Tunicamycin treatment (Fig. S1L) SMPD2 KD-downregulated LAMP1 protein level did not recover under $4 \mu 8 \mathrm{C}$ treatment. These data indicate that reduced mRNA level upon SMPD2 KD is not caused by degradation through the RIDD pathway.

Since SMPD2 KD cells failed to activate an efficient UPR signaling upon ER stress induction and are less viable under normal conditions, we next analyzed how SMPD2 KD affects cellular fitness under ER stress. Indeed, SMPD2 KD cells are less viable upon ER stress than their control counterparts; especially, SMPD2 KD cells treated with thapsigargin are significantly less viable than SMPD2 KD treated with DMSO. Surprisingly, $4 \mu 8 \mathrm{C}$ significantly increased viability in both control and SMPD2 KD cells (Fig. 2N). In summary, the above data suggest that although SMPD2 KD does not downregulate LAMP1 mRNA through the RIDD pathway and does not induce ER stress, a full-potential UPR signaling activation upon ER stress is not achieved in SMPD2 KD cells, and consequently their cellular fitness is impaired under ER stress conditions. 


\section{SMPD2 KD arrests cells in the G1 phase}

As SMPD2 KD significantly decreased cell viability (Fig. S1A, 2N), and JNK signaling was shown to induce apoptosis via nSMase1-induced ceramide generation under various stress conditions (Yabu et al, 2015), we next investigated whether SMPD2 KD induces apoptosis using flow cytometry (Fig. S2A). In alignment with the previous results (Fig. S1A, 2N), SMPD2 KD significantly reduced viable cells in an Annexin/PI assay (Fig. 3A). Although early apoptosis was significantly increased $\left(\mathrm{A}^{-} / \mathrm{PI}^{+}\right)$, no significant change in late apoptosis $\left(\mathrm{A}^{+} / \mathrm{PI}^{+}\right)$and necrosis levels $\left(\mathrm{A}^{-} / \mathrm{PI}^{+}\right)$were observed upon SMPD2 KD (Fig. 3A). Concordantly, SMPD2 KD did not induce the cleavage of different apoptosis marker proteins as determined by western blot analysis of Caspase-3, -7, and PARP (Fig. 3B, Fig.S2B-E).

Since the reduced cell viability upon SMPD2 KD is not caused by apoptosis, we next analyzed the cell proliferation rate by incucyte imaging. Indeed, the cell proliferation rate was significantly lowered by SMPD2 KD (Fig. 3C). Additionally, SMPD2 KD cells are significantly bigger when compared to the control cells (Fig. 3D, Fig. S2F), suggesting an SMPD2 KD-induced cell cycle delay. Cell cycle progression analysis by flow cytometry (Fig. S2G) revealed SMPD2 KD to significantly increase the percentage of cells in G1 (Fig. 3E). The percentage of cells in G1 was furthermore increased (by around $20 \%$ ) when SMPD2 KD duration was increased from $48 \mathrm{~h}$ to $72 \mathrm{~h}$ (Fig. 3F) and reduced slightly after $96 \mathrm{~h}$ when compared to the $72 \mathrm{~h}$ SMPD2 KD (Fig. S2H). Overall, these data indicate that a significant number of SMPD2 KD cells show a delay in cell cycle progression. To test whether SMPD2 KD causes a transient G1 arrest, we analyzed the protein expression of two G1 cell cycle arrest markers - P21 and P27, along with P53, which regulate these two proteins (Chen, 2016). SMPD2 KD significantly upregulated the protein expression of both P21 and P27 (Fig. 3G, H), while P53 protein levels remained unchanged upon SMPD2 KD (Fig. S3A). In agreement with the FACS data (Fig. 3F), both P21 and P27 protein expression reached a maximum after $72 \mathrm{~h}$ of SMPD2 KD (Fig. 3G, H) when compared to $96 \mathrm{~h}$ SMPD2 KD (Fig. S3B, C). In summary, these data indicate that SMPD2 KD cells are bigger and proliferate slower than the control cells, as they are temporarily arrested in the G1 phase of the cell cycle at the peak of the transient SMPD2 KD. 


\section{SMPD2 KD downregulates the Wnt signaling pathway}

Next, we investigated molecular pathways through which SMPD2 KD causes G1 cell cycle arrest. Since various lipids in the nucleus are indicated to regulate many processes including gene transcription (Martelli et al, 2004), we analyzed if SMPD2 KD upregulates P21 and P27 for G1 arrest through the P53-dependent DNA damage response pathway. DNA damage, through ataxia telangiectasia mutated (ATM) or Rad3-related protein (ATR), phosphorylates two checkpoint kinases (Chk1 and Chk2), which then activates P53 by phosphorylation (Chen, 2016) (Fig. 4A). Activated P53 upregulates, among many of its target genes, two cell cycle inhibitory proteins P21 and P27. Therefore, an increase in phosphorylated Chk1 and Chk2 could indicate activation of the DNA damage response pathway upon SMPD2 KD. The expression level of the phosphorylated Chk1 and phosphorylated $\mathrm{Rb}$ (Retinoblastoma protein), another cell cycle inhibitory protein, was not changed by SMPD2 KD (Fig. 4B, C; Fig. S3D). However, SMPD2 KD significantly decreased the phosphorylated Chk2 level (Fig. 4B, C), in line with a role of Chk2 at the crossroad of DNA damage and metabolic fitness (Ajazi et al, 2021).

Extracellular growth signals are integrated into intracellular signals by pathways such as PI3K/Akt to drive cell cycle progression (Vivanco \& Sawyers, 2002) (Fig. 4D). SMPD2 was shown to exhibit a negative gene interaction with PTEN (phosphatase and tensin Homolog), a tumor suppressor gene that inhibits G1-S transition by antagonizing PI3k/Akt pathway activation (Fig. 4D) (Vizeacoumar et al, 2013). Therefore, we analyzed the level of phosphorylated PTEN and AKT upon SMPD2 KD. Although phosphorylated PTEN levels remained unchanged, SMPD2 KD significantly reduced the level of phosphorylated Akt (Fig. 4E, F) in line with a role of SMPD2 KD in G1-S transition through the PI3K/Akt pathway.

Wnt signaling is another major pathway that drives cell proliferation (Freese et al, 2010). Interestingly, SMPD2 KD reduced basal Wnt activity, but also Wnt activity induced by $\beta$ catenin accumulation through APC KD (Fig. 4G). Reduction of APC KD-induced Wnt activity by SMPD2 KD indicates that the KD affects the Wnt signaling at the level or downstream of $\beta$-catenin. Indeed, $\beta$-catenin protein levels were downregulated by SMPD2 KD in HeLa cells (Fig. S3E, F). Surprisingly, in HCT116 cells, although $\beta$-catenin protein levels remained unchanged, Wnt3a protein levels were significantly reduced by 
SMPD2 KD (Fig. 4H, I). We furthermore analyzed Wnt signaling components upon SMPD2 KD by qPCR in HCT116. In alignment with the reduction in Wnt3a protein levels, SMPD2 KD dramatically reduced Wnt3a mRNA (Fig. 4J) and significantly upregulated AXIN2 mRNA (Fig. 4J), which is involved in the negative feedback loop of Wnt signaling (Lustig et al, 2001). The mRNA levels of the other Wnt signaling components CTNNB-1, EVI, and WNT7B remained unchanged upon SMPD2 KD (Fig. S3G). Additionally, SMPD4, another member of nSMases, showed no compensatory increase in mRNA levels upon SMPD2 KD (Fig. S3H). Since many proteins and mRNA are downregulated upon SMPD2 KD, we next analyzed the overall protein translation upon SMPD2 KD by puromycin assay (Goodman \& Hornberger, 2013) and using protein translation inhibitor cycloheximide $(\mathrm{CHX})$ as a negative control. Indeed, SMPD2 KD significantly reduced the overall protein translation (Fig. $4 \mathrm{~K}, \mathrm{~L}$ ).

In summary, the data above indicate that SMPD2 KD seems to affect many cellular processes by downregulating their signaling components including Wnt signaling - which could explain the reduction in global protein translation and $\mathrm{G} 1$ cell cycle arrest.

\section{Discussion}

We found that nSMase 1 plays an important role in maintaining cellular fitness. NSMase1 is required for activating a full-potential adaptive UPR upon ER stress induction with tunicamycin and thapsigargin- two inhibitors that cause ER stress through distinct mechanisms. SMPD2 KD cells failed to upregulate multiple genes that are usually upregulated through UPR pathways upon ER stress to the same levels as their control counterparts. Furthermore, we show that nSMase1 is necessary for proper cell cycle progression during the $\mathrm{G} 1$ phase as SMPD2 KD led to a significant G1 arrest. Moreover, the overall protein translation at the ribosome was reduced by SMPD2 KD. These data, for the first time, establish an important biological role for SMPD2 at the level of UPR activation upon ER stress and cell cycle progression.

We further show that nSMase1 regulates LAMP1 protein expression at the level of its mRNA but not LAMP2. LAMP1 and LAMP2 are the major glycoproteins localized on the lysosomal membrane and thought to contribute to lysosomal integrity, catabolism, and pH maintenance (Sawada et al, 1993). SMPD2 KD downregulated LAMP1 mRNA and 
thereby reduced its protein expression. Interestingly, lysosomal acidification and function remain unaffected by this significant reduction in the LAMP1 protein levels as both lysotracker staining and the autophagy flux remained unchanged. Therefore, the downstream biological implication of this dramatic LAMP1 downregulation upon SMPD2 KD would be of great interest for future studies.

ER stress is sensed by three ER membrane proteins - IRE1 $\alpha$, PERK, and ATF6a - which initiate UPR through three distinct pathways by upregulating different proteins (Hetz et al, 2020). Although nSMase1 expression was increased by ER stress, SMPD2 KD alone did not induce an ER stress condition as no UPR activation was observed. Upon ER stress induction, SMPD2 KD cells failed to upregulate multiple genes under the control of all three master regulators. These data indicate that SMPD2 KD affects all the three UPR pathways in the ER. Indeed, several early studies showed that overexpressed nSMase1 resides in the ER (Fensome et al, 2000; Rodrigues-Lima et al, 2000; Tomiuk et al, 2000) and recent affinity-based mass spectrometry studies showed that nSMase 1 interacts with multiple ER-resident proteins including reticulon 3 and reticulon 4 (Huttlin et al, 2017, 2021). Therefore, based on the observations from our study, it is clear that nSMase1 plays a functional role in restoring ER function and homeostasis under ER stress conditions. The UPR signaling activation persists until an adaptive response to the ER stress is achieved, in the case of unresolvable stress, the UPR turns pro-apoptotic to induce cell death (Hetz et al, 2020). Indeed, due to their inability to mount a full-potential UPR signaling, SMPD2 KD cells are less viable upon ER stress induction.

Additionally, SMPD2 KD significantly downregulated LAMP1 and Wnt3a mRNAs - both of which are translated and subsequently modified in the ER as an integral membrane and a secretory protein respectively. NSMase1 could affect signaling recognition particle (SRP)-dependent targeting of these proteins to the ER for translation as nSMase1 was shown to interact with both signal recognition particle receptor B (SRPRB) and the translocon-associated signal sequence receptor 2 (SSR2). These two proteins are ER membrane proteins required for proper docking of the whole mRNA:ribosome:polypeptide:SRP complex to the ER for efficient translation (Akopian et al, 2013). It is therefore conceivable that nSMase1 plays an important role in the translation of these proteins possibly by providing specific lipids to establish membrane 
nanodomains for efficient docking of this co-translation machinery to the ER. In this way, downregulation of LAMP1 and Wnt3a could be due to a disrupted SRP-dependent targeting of these mRNAs to the ER for translation upon SMPD2 KD. In line with its ER localization and potential functions, nSMase1 also interacts with several GPI (Glycosylphosphatidylinositol) anchor attaching proteins such as glycosylphosphatidylinositol anchor attachment 1 (GPAA1) and Phosphatidylinositol glycan anchor biosynthesis, class S (PIG-S) (Huttlin et al, 2021).

Overall, the data from others and our study demonstrate that nSMase1 at the ER plays an important role in activating an efficient adaptive UPR upon ER stress and could also regulate other aspects of $\mathrm{ER}$ function such as translation and post-translational modifications.

Since SMPD2 KD cells are arrested in the G1 phase with both P21 and P27 upregulated, it is possible that nSMase1 activity, by generating required structural lipids, might drive the remodeling of the nuclear envelope, chromatin, and nuclear matrix during cell cycle progression (Mizutani et al, 2001). Alternatively, since nSMase1 belongs to the superfamily of endo/exonucleases (Bill X.Wu, Christopher J.Clarke, 2010) that cleave the phosphodiester bond between the successive nucleotides, it may hydrolyze the phosphodiesterase bond of the DNA. As described for many endo/exonucleases (Nishino \& Morikawa, 2002), it, therefore, could be involved in DNA repair and cell cycle progression. In this way, inefficient DNA repair upon SMPD2 KD might induce a DNAdamage-like response that upregulates P21 and P27 to induce G1 cell cycle arrest. Therefore, it is noteworthy to further investigate the activation of tumor suppressor gene P53 upon SMPD2 KD. P53 senses DNA damage and mediates cell cycle arrest by upregulating P21 and P27 (Chen, 2016).

Duplication of not only cellular DNA content but also of membranes and organelles is a prerequisite for cellular growth and division. Lipids - both storage and membrane - play an important role in cell cycle regulation (Storck et al, 2018). For example, two triacylglycerol lipases Tgl3 and Tgl4 are required for efficient cell cycle progression during the G1/S transition in yeast cells (Chauhan et al, 2015). Specifically, lipolysis-derived sphingolipids activate PP2A, a major cell cycle regulating protein phosphatase, to dephosphorylate SWE1 (human ortholog WEE1) for efficient cell cycle progression 
(Chauhan et al, 2015). Therefore, it is conceivable that ceramide generated by nSMase 1 acts as a second messenger targeting proteins involved in cell-cycle regulation such as PP2A. Indeed, several studies proposed PP1 and PP2A as the potential intracellular protein target of ceramide. PP2A dephosphorylates over 300 substrates involved in the cell cycle, thereby regulating almost all the major pathways including the Wnt pathway and the cell cycle checkpoints (Wlodarchak \& Xing, 2016). Therefore, reduced Wntsignaling could be also due to deregulated PP2A activity upon SMPD2 KD. Collectively, these data suggest that nSMase1 plays an important role in overall cellular fitness and proliferation without which cells enter a transient G1 arrest with reduced global protein translation.

So far, the biological role as well the physiological substrate for nSMase1 is unknown. Our study shows that nSMase1 plays a vital role in overall cellular fitness and survival as SMPD2 KD induced transient G1 arrest. Additionally, nSMase1 activity is essential for activating an efficient UPR upon ER stress induction. The observations and data described herein would serve as a strong foothold for further studies in unraveling not only the molecular function but also the molecular pathways through which nSMase 1 contributes to maintaining cellular homeostasis.

\section{Materials and methods}

\section{Cell culture and transfection (siRNA and plasmids)}

HeLa (kindly provided by Holger Bastians, Goettingen) and HCT116 cells (DSMZ) were maintained in DMEM (Thermo Scientific Life technologies) supplemented with $10 \%$ fetal calf serum (Biochrom) and $10 \mu \mathrm{g} / \mathrm{ml}$ Penicillin/Streptomycin (Sigma-Aldrich) at $37^{\circ} \mathrm{C}$ in a humidified atmosphere with $5 \% \mathrm{CO}_{2}$. Cells were transiently transfected with Screenfect siRNA (Dharmacon) for indicated duration according to the manufacturer's instructions. The cells were authenticated and routinely checked for mycoplasma contamination. Cells were treated with the drugs in the following concentrations for indicated durations. Bafilomycin (Sigma-Aldrich, 100ng/ml, 16 h); MG132 (Sigma-Aldrich, 100ng/ml, 16 h); $4 \mu 8 \mathrm{C}$ (Millipore Merck, $49 \mu \mathrm{M}$, Duration as indicated); Cycloheximide (Carlroth, $20 \mu \mathrm{g} / \mathrm{ml}$, $16 \mathrm{~h}$ ). 
Table 1: Dharmacon siRNA SMARTpools against the indicated genes used in the study

\begin{tabular}{|c|c|c|}
\hline Target Gene & Catalog & Sequence \\
\hline & Number & \\
\hline \multirow[t]{4}{*}{ SMPD2 } & D-006677-01 & GCAGAGAGGUCGCCGUUGA \\
\hline & D-006677-02 & GGAGGUCAAUGGCUUAUAU \\
\hline & D-006677-03 & CAAGGCAGUUUCUGGGUUU \\
\hline & D-006677-04 & UGAAACCACUACAGGCUUU \\
\hline \multirow[t]{3}{*}{ APC } & D-003869-05 & GAUGAUAUGUCGCGAACUU \\
\hline & D-003869-06 & GAGAAUACGUCCACACCUU \\
\hline & D-003869-07 & GAACUAGAUACACCAAUAA \\
\hline \multirow[t]{4}{*}{ EVI/ WLS } & D-018728-01 & ACGAAUCCCUUCUACAGUA \\
\hline & D-018728-02 & UAACGGAAGGCCAUUGGAA \\
\hline & D-018728-03 & UAAAGGAUAUCCGGUUGGU \\
\hline & D-018728-04 & GAACCACAUCGCAGGGUAU \\
\hline SDCBP & D-008270-01 & GCAAGACCUUCCAGUAUAA \\
\hline & D-008270-02 & UAACAUCCAUAGUGAAAGA \\
\hline
\end{tabular}


D-008270-03

D-008270-04

NSMAF

D-017920-01

D-017920-02

D-017920-03

D-017920-04

Non-targeting siRNA

D-001210-03
GAAGGACUCUCAAAUUGCA

GGAUGGAGCUCUGAUAAAG

CGAGUGGAUUGAUCUAAUA

UUACGCAAAUCUUGGAAUU

CGCAAUGGAUCUUCAGUAU

CGACAUCUACCAAAAGUUC

AUGUAUUGGCCUGUAUUA G

\section{Antibodies}

The following antibodies and dilutions were used for western blot (WB) or for immunofluorescence staining (IF): LAMP1 (WB 1:1000, Abcam, ab24170); Syntenin (WB 1:1000, Abcam, ab133267); GAPDH (WB 1:1000, Millipore, CB1001); Calnexin (WB 1:1000, Abcam, ab75801), P62 (WB 1:1000, Abnova); LC3B (WB 1:1000, Santa Cruz); Ceramide (IF 1:100, Enzo, ALX-804-196); BiP (WB 1:1000, CST), nSMase1 (IF 1:100, WB 1:1000, Sigma); $\beta$-Catenin (WB 1:1000, BD Transduction), PDI (WB 1:1000, CST), FL-Caspase 3 (WB 1:1000, CST); C-Caspase-3 (WB 1:100, CST), FL-Caspase 7 (WB 1:1000, CST); C-Caspase-7 (WB 1:1000); FL-PARP (WB 1:1000, CST), C-PARP (WB 1:1000, CST), pCHk2 (Thr68) (WB 1:1000, CST); p-Rb (Ser807/811) (WB 1:1000, CST); p-Chk1 (Ser345) (WB 1:1000, CST); p-AKT (Ser473) (WB 1:1000, CST); Puromycin (WB 1:1000, Millipore);; pPTEN (Ser380) (WB 1:1000, CST) 


\section{Western blot analysis}

Cell lysates in the SDS-PAGE sample buffer were boiled for $5 \mathrm{~min}$ before separating the protein on $4-12 \%$ gradient gels (Bolt Bis-Tris Plus Gels, Thermo Scientific). Proteins were then transferred onto PVDF membranes (Merck) and blocked with $5 \%$ (wt/vol) milk-TBST for $30 \mathrm{~min}$ before incubating with primary antibodies overnight at $4^{\circ} \mathrm{C}$. After washing, membranes were incubated with fluorescently labeled secondary antibodies at room temperature in the dark and detected using the Li-COR Odyssey system. Western blot quantifications were done with Odyssey Infrared Image Studio Lite Ver 5.2

\section{Autophagy flux measurement}

Densitometric analysis of the LC3B-II, P62, and GAPDH bands was performed using Odyssey Infrared Image Studio Lite Ver 5.2. To determine autophagy flux, under both basal and serum-starvation autophagy induced conditions, lysosomal turnover of endogenous LC3B-II and P62 were determined. LC3B-II and P62 bands were normalized to GAPDH (LC3B-II/GAPDH, P62/GAPDH). Autophagy flux was then determined by dividing the normalized value of the Bafilomycin-treated lysate by the normalized value of the control-treated lysate of the same sample(Tanida et al, 2005).

\section{Immunostaining, microscopy, and image analysis}

Cells were grown in 8-well microscopic coverslips (Sarstedt and IBIDI for live imaging), reverse transfected with indicated siRNAs, and were fixed and permeabilized with $4 \%$ paraformaldehyde and $0.2 \%$ Triton-X-100. The slides were then blocked in $3 \%$ bovine serum albumin diluted in PBS, followed by 90 min incubation with primary antibodies. After washing three times, cells were incubated with secondary antibodies conjugated to Alexa-Fluor-488 or 546. Nuclei and actin were labeled by Hoechst and conjugated phalloidin respectively. $200 \mathrm{nM}$ ER tracker (Thermo Scientific Life technologies, E34250) were added to live cells. The cells were visualized with a Zeiss LSM780 confocal microscope (Plan Neofluor 63X/oil NA 1.4 objective). Staining and microscopy conditions were kept identical for comparisons. The number of puncta/cell quantifications were performed using available pipelines with some modifications in CellProfiler (Broad Institute of MIT and Harvard). 


\section{CellTiter-Glo® luminescent viability assay}

Cell viability was measured by performing a CellTiter-Glo assay (Promega, G8461). Cells were seeded in a 96-well plate. After reverse siRNA transfection or drug treatment for the indicated duration, $100 \mu \mathrm{l}$ of the cell titer glow reagent (Promega) diluted to 1:5 with PBS were added to each well. The plate was incubated on a shaker for 2 min at RT to allow cell lysis and then incubated without shaking for $10 \mathrm{~min}$ at RT to allow luminescence signal stabilization. The signal was measured using a Centro LB 960 microplate luminometer (Berthold Technologies) and data were analyzed using MikroWin 2000 lite Version 4.43.

\section{Incucyte proliferation assay}

HeLa cells transfected with control or SMPD2 siRNA for $48 \mathrm{~h}$ were replated and 6000 transfected cells per well were seeded into Incucyte® Imagelock 96-well plates. The realtime proliferation of the cells was detected by the Incucyte ${ }^{\circledR}$ S2 Live Cell Analysis System (Sartorious) by imaging each well at $2 \mathrm{~h}$ intervals.

\section{RNA isolation and real-time qPCR}

Total cellular RNA was isolated using Trizol (Thermo Scientific Invitrogen). The reverse transcription was carried out with $2 \mu \mathrm{g}$ of RNA. The resulting cDNA product was analyzed by real-time quantitative PCR using Taq Universal SYBRgreen Supermix (Bio-Rad). Transcript Ct-values were converted to fold change expression changes (2- $\Delta \Delta \mathrm{Ct}$ values) after normalization to the housekeeping gene $\beta$-actin. Quantitative real-time PCR was performed using a CFX system (Bio-Rad).

\section{Table 2: List of primers with their respective sequences used in the study}

\begin{tabular}{cll}
\hline Gene & Company & \\
& & \multicolumn{1}{c}{ Sequence (5'-3') } \\
\hline SMPD2 & Merck Millipore & FP: TTTGCTGGAGGAGGTGTGGAG \\
& & RP: AAGCTCCTGGATTGGATGTTTGG \\
LAMP1 & Merck Millipore & FP: CAGTTCGGGATGAATGCAAG \\
& & RP: TGCACTTGTAGGAATTGCCG \\
CHOP & Merck Millipore & FP: AGAACCAGGAAACGGAAACAGA
\end{tabular}


RP: TCTCCTTCATGCGCTGCTTT

$\begin{array}{cll}\text { EDEM } & \text { Merck Millipore } & \text { FP: CAAGTGTGGGTACGCCACG } \\ \text { BiP } & \text { Rerck Millipore } & \text { FP: TGTTCAACCAATTATCAGCAAACTC } \\ & & \text { RP: TTCTGCTGTATCCTCTTCACCAGT } \\ \text { Calnexin } & \text { Merck Millipore } & \text { FP: TTCACATAGGCACCACCACA } \\ & & \text { RP: GGAAGTGGTTGCTGTGTATG } \\ \text { CTNNB-1 } & \text { Merck Millipore } & \text { FP: AAGCGGCTGTTAGTCACTGG } \\ & & \text { RP: CGAGTCATTGCATACTGTCCAT } \\ \text { sXBP-1 } & \text { Merck Millipore } & \text { FP:C TGAGTCCGAATCAGGTGCAG } \\ & & \text { RP: ATCCATGGGGAGATGTTCTGG } \\ \text { WNT3A } & \text { Merck Millipore } & \text { FP: AGCAGGACTCCCACCTAAAC RP } \\ & & \text { RP: AGAGGAGACACTAGCTCCAGG } \\ \text { WNT7B } & \text { Merck Millipore } & \text { FP: CCCGGCAAGTTCTCTTTCTTC } \\ & & \text { RP: GGCGTAGCTTTTCTGTGTCCAT } \\ \text { B-actin } & \text { Merck Millipore } & \text { FP: GAGCACAGAGCCTCGCCTTT } \\ & & \text { RP: ACATGCCGGAGCCGTTGTC } \\ \text { ATF4 } & \text { Merck Millipore } & \text { FP: GTTCTCCAGCGACAAGGCTA } \\ & & \text { RP: ATCCTGCTTGCTGTTGTTGG } \\ \text { SMPD3 } & \text { Merck Millipore } & \text { FP: GTGTAACGACGATGCCCTGG } \\ & & \text { RP: GGATGGCGCTGTCCTCTTG } \\ & & \end{array}$

\section{Wnt reporter activity assay}

Endogenous Wnt signaling activity was assessed by employing a dual-luciferase $\beta$ catenin/TCF4 reporter assay. HCT116 cells were transfected with a TCF firefly reporter plasmid (pgGL4.1-6xKD FLuc) (Demir et al, 2013) and a constitutively $\beta$-catenin renilla reporter plasmid (pgGL4.1-RLuc) (Demir et al, 2013) $24 \mathrm{~h}$ after reverse transfection with the indicated siRNA. Relative Wnt reporter activity was determined by normalizing the firefly luminescence value to the renilla luminescence value after $48 \mathrm{~h}$ of the plasmid transfection. 


\section{Cell cycle analysis by flow cytometry}

Flow cytometry was used to analyze cell cycle distribution. Trypsinized cells pelleted at $400 \mathrm{~g}$ for 5 min were fixed by adding cold $70 \%$ ethanol dropwise while vortexing. Fixed cells were incubated at $-20^{\circ} \mathrm{C}$ for $1 \mathrm{~h}$ before pelleting at $400 \mathrm{~g}$ for $5 \mathrm{~min}$ and washed once in PBS. Mitotic cells were labeled by staining with primary antibody against phosphoMpm-2 (1:1600) for $1 \mathrm{hr}$ followed by 30 min incubation in secondary antibody conjugated to Alexa Fluor 488 (1:2000). Cells were then centrifuged at $400 \mathrm{~g}$ for $5 \mathrm{~min}$ and resuspended in PBS with $0.5 \mu \mathrm{g} / \mathrm{ml}$ RNAseA and $10 \mu \mathrm{g} / \mathrm{ml}$ propidium iodide to stain for DNA content. Cell cycle distribution of 10000 cells was analyzed by using a BD FACSCanto II (BD Biosciences) and BD FACSDiva 9.0.1 software.

\section{Apoptosis analysis by flow cytometry}

For apoptosis analysis, FITC Annexin V Apoptosis Detection Kit with PI (BioLegend) was used according to the manufacturer's instructions. Briefly, cells were stained with Annexin-FITC and propidium iodide for $15 \mathrm{~min}$ at RT after washing the cells once with PBS and then resuspending in Annexin V binding buffer. Dead and Floating cells were added to each sample for analysis by pelleting the conditioned medium at $1500 \mathrm{~g}$ for 5 min. Apoptosis of 10000 cells from each sample was analyzed by using a BD FACSCanto II (BD Biosciences) and BD FACSDiva 9.0.1 software.

\section{Puromycin assay}

To analyze protein translation rate, $16 \mu \mathrm{g} / \mathrm{ml}$ of puromycin were added to siRNA reverse transfected or inhibitor-treated cells as indicated for $45 \mathrm{~min}$ (Goodman \& Hornberger, 2013). Cell extracts were harvested in RIPA lysis buffer and puromycinylated proteins were detected by western blot using puromycin antibody.

\section{Statistics}

Data were analyzed using GraphPad Prism 6 built-in tests. All data are presented as means \pm S.D.s. Details about the significance test, the number of replicates, and the $P$ values are reported in the respective figure legends.

\section{Acknowledgments}

The authors would like to thank AG Bastians (University of Goettingen) for HeLa cells. We thank Dr. Karen Linnenmannstöns, Dr.Leonie Witte, and Dr. Pradhipa Karuna M for 
critically reading the manuscript and Mona Honemann-Capito for her excellent technical and scientific support.

\section{Author contribution}

D.C.: Conceptualization, investigation, formal analysis, writing - original draft, review \& editing J.C.G.: Conceptualization, Writing - review \& editing, funding acquisition

\section{Conflict of Interest}

The authors declare no conflict of interests

\section{References}

Airola M V \& Hannun YA (2013) Sphingolipid metabolism and neutral sphingomyelinases. Handb Exp Pharmacol (215): 57-

Ajazi A, Bruhn C, Shubassi G, Lucca C, Ferrari E, Cattaneo A, Bachi A, Manfrini N, Biffo S, Martini E, et al (2021) Endosomal trafficking and DNA damage checkpoint kinases dictate survival to replication stress by regulating amino acid uptake and protein synthesis. Dev Cell 56: 2607-2622.e6

Akopian D, Shen K, Zhang X \& Shan SO (2013) Signal recognition particle: An essential protein-targeting machine

Baietti MF, Zhang Z, Mortier E, Melchior A, Degeest G, Geeraerts A, Ivarsson Y, Depoortere F, Coomans C, Vermeiren E, et al (2012) Syndecan-syntenin-ALIX regulates the biogenesis of exosomes. Nat Cell Biol 14: 677-685

Bill X.Wu, Christopher J.Clarke and YAH (2010) Mammalian

Neutralsphingomyelinases: Regulation and Roles in Cell Signaling Responses.

Neuromolecular Med 12: 320-330

Chauhan N, Visram M, Cristobal-Sarramian A, Sarkleti F \& Kohlwein SD (2015)

Morphogenesis checkpoint kinase Swe1 is the executor of lipolysis-dependent cellcycle progression. Proc Natl Acad Sci U S A 112: E1077-E1085

Chen J (2016) The cell-cycle arrest and apoptotic and progression. Cold Spring Harb

Perspect Biol: 1-16

Choezom D \& Gross JC (2022) Neutral Sphingomyelinase 2 controls exosomes secretion via counteracting V-ATPase-mediated endosome acidification. J Cell Sci 
Cross BCS, Bond PJ, Sadowski PG, Jha BK, Zak J, Goodman JM, Silverman RH, Neubert TA, Baxendale IR, Ron D, et al (2012) The molecular basis for selective inhibition of unconventional mRNA splicing by an IRE1-binding small molecule.

Proc Natl Acad Sci U S A 109: 869-878

Demir K, Kirsch N, Beretta CA, Erdmann G, Ingelfinger D, Moro E, Argenton F, Carl M, Niehrs C \& Boutros M (2013) RAB8B Is Required for Activity and Caveolar Endocytosis of LRP6. Cell Rep 4: 1224-1234

Fensome AC, Rodrigues-Lima F, Josephs M, Paterson HF \& Katan M (2000) A neutral magnesium-dependent sphingomyelinase isoform associated with intracellular membranes and reversibly inhibited by reactive oxygen species. J Biol Chem 275: $1128-1136$

Freese JL, Pino D \& Pleasure SJ (2010) Wnt signaling in development and disease.

Neurobiol Dis 38: 148-153

Goodman CA \& Hornberger TA (2013) Measuring protein synthesis with SUnSET: A valid alternative to traditional techniques? Exerc Sport Sci Rev 41: 107-115

Hannun YA \& Obeid LM (2008) Principles of bioactive lipid signalling: lessons from sphingolipids. Nat Rev Mol Cell Biol 9

Hetz C, Zhang K \& Kaufman RJ (2020) Mechanisms, regulation and functions of the unfolded protein response. Nat Rev Mol Cell Biol 21: 421-438

Hofmann K, Tomiuk S, Wolff G \& Stoffel W (2000) Cloning and characterization of the mammalian brain-specific, Mg2+-dependent neutral sphingomyelinase. Proc Natl Acad Sci U S A 97: 5895-900

Hollien J, Lin JH, Li H, Stevens N, Walter P \& Weissman JS (2009) Regulated Ire1dependent decay of messenger RNAs in mammalian cells. J Cell Biol 186: 323331

Huttlin EL, Bruckner RJ, Navarrete-Perea J, Cannon JR, Baltier K, Gebreab F, Gygi MP, Thornock A, Zarraga G, Tam S, et al (2021) Dual proteome-scale networks reveal cell-specific remodeling of the human interactome. Cell 184: 3022-3040.e28 Huttlin EL, Bruckner RJ, Paulo JA, Cannon JR, Ting L, Baltier K, Colby G, Gebreab F, Gygi MP, Parzen H, et al (2017) Architecture of the human interactome defines 
protein communities and disease networks. Nature 545: 505-509

Jaffrézou JP, Levade T, Bettaïeb A, Andrieu N, Bezombes C, Maestre N, Vermeersch

S, Rousse A \& Laurent G (1996) Daunorubicin-induced apoptosis: Triggering of ceramide generation through sphingomyelin hydrolysis. EMBO J 15: 2417-2424

Jana A \& Pahan K (2004) Fibrillar amyloid- $\beta$ peptides kill human primary neurons via

NADPH oxidase-mediated activation of neutral sphingomyelinase: Implications for

Alzheimer's disease. J Biol Chem 279: 51451-51459

Lee JT, Xu J, Lee JM, Ku G, Han X, Yang DI, Chen S \& Hsu CY (2004) Amyloid- $\beta$

peptide induces oligodendrocyte death by activating the neutral sphingomyelinaseceramide pathway. J Cell Biol 164: 123-131

Lustig B, Jerchow B, Sachs M, Weiler S, Pietsch T, Rarsten U, Van De Wetering M, Clevers H, Schlag PM, Birchmeier W, et al (2001) Negative feedback loop of Wnt signaling through upregulation of conductin/axin2 in colorectal and liver tumors.

Langenbeck's Arch Surg 386: 466

Martelli AM, Falà F, Faenza I, Billi AM, Cappellini A, Manzoli L \& Cocco L (2004) Metabolism and signaling activities of nuclear lipids. Cell Mol Life Sci 61: 11431156

Maurel M, Chevet E, Tavernier J \& Gerlo S (2014) Getting RIDD of RNA: IRE1 in cell fate regulation. Trends Biochem Sci 39: 245-254

Mizutani Y, Tamiya-Koizumi K, Nakamura N, Kobayashi M, Hirabayashi Y \& Yoshida S (2001) Nuclear localization of neutral sphingomyelinase 1: biochemical and immunocytochemical analyses. J Cell Sci 114: 3727-3736

Nishino T \& Morikawa K (2002) Structure and function of nucleases in DNA repair:

Shape, grip and blade of the DNA scissors. Oncogene 21: 9022-9032

Oslowski CM \& Urano F (2011) Measuring ER stress and the unfolded protein response using mammalian tissue culture system 1 st ed. Elsevier Inc.

Parashuraman S \& D'Angelo G (2019) Visualizing sphingolipid biosynthesis in cells.

Chem Phys Lipids 218: 103-111

Philipp S, Puchert M, Adam-Klages S, Tchikov V, Winoto-Morbach S, Mathieu S,

Deerberg A, Kolker L, Marchesini N, Kabelitz D, et al (2010) The Polycomb group 
protein EED couples TNF receptor 1 to neutral sphingomyelinase. Proc Natl Acad Sci U S A 107: 1112-1117

Rodrigues-Lima F, Fensome AC, Josephs M, Evans J, Veldman RJ \& Katan M (2000)

Structural requirements for catalysis and membrane targeting of mammalian enzymes with neutral sphingomyelinase and lysophospholipid phospholipase C activities: Analysis by chemical modification and site-directed mutagenesis. J Biol Chem 275: 28316-28325

Sawada R, Jardine KA \& Fukuda M (1993) The genes of major lysosomal membrane glycoproteins, lamp-1 and lamp-2. 5'-Flanking sequence of lamp-2 gene and comparison of exon organization in two genes. J Biol Chem 268: 9014-9022

Sawai H, Domae N, Nagan N \& Hannun YA (1999) Function of the cloned putative neutral sphingomyelinase as lyso-platelet activating factor-phospholipase C. J Biol Chem 274: 38131-38139

Schwarz DS \& Blower MD (2016) The endoplasmic reticulum: Structure, function and response to cellular signaling. Cell Mol Life Sci 73: 79-94

Storck EM, Özbalci C \& Eggert US (2018) Lipid Cell Biology: A Focus on Lipids in Cell Division. Annu Rev Biochem 87: 839-869

Tanida I, Minematsu-Ikeguchi N, Ueno T \& Kominami E (2005) Lysosomal turnover, but not a cellular level, of endogenous LC3 is a marker for autophagy. Autophagy 1: 84-91

Tomiuk S, Hofmann K, Nix M, Zumbansen M \& Stoffel W (1998) Cloned mammalian neutral sphingomyelinase: Functions in sphingolipid signaling? Proc Natl Acad Sci 95: 3638-3643

Tomiuk S, Zumbansen M \& Stoffel W (2000) Characterization and subcellular localization of murine and human magnesium-dependent neutral sphingomyelinase. J Biol Chem 275: 5710-5717

Tonnetti L, Verí MC, Bonvini E \& D’Adamio L (1999) A role for neutral sphingomyelinase-mediated ceramide production in $\mathrm{T}$ cell receptor-induced apoptosis and mitogen-activated protein kinase-mediated signal transduction. $J$ Exp Med 189: 1581-9 
Vielhaber G, Brade L, Lindner B, Pfeiffer S, Wepf R, Hintze U, Wittern KP \& Brade H (2001) Mouse anti-ceramide antiserum: A specific tool for the detection of endogenous ceramide. Glycobiology 11: 451-457

Vivanco I \& Sawyers CL (2002) The phosphatidylinositol 3-kinase-AKT pathway in humancancer. Nat Rev Cancer 2: 489-501

Vizeacoumar FJ, Arnold R, Vizeacoumar FS, Chandrashekhar M, Buzina A, Young JTF, Kwan JHM, Sayad A, Mero P, Lawo S, et al (2013) A negative genetic interaction map in isogenic cancer cell lines reveals cancer cell vulnerabilities. Mol Syst Biol 9

Wlodarchak N \& Xing Y (2016) PP2A as a master regulator of the cell cycleThe Impact of Pollution on Worker Productivity. Crit Rev Biochem Mol Biol 51: 162-184

Wu BX, Rajagopalan V, Roddy PL, Clarke CJ \& Hannun YA (2010) Identification and characterization of murine mitochondria-associated neutral sphingomyelinase (MAnSMase), the mammalian sphingomyelin phosphodiesterase 5. J Biol Chem 285: 17993-18002

Yabu T, Shiba H, Shibasaki Y, Nakanishi T, Imamura S, Touhata K \& Yamashita M (2015) Stress-induced ceramide generation and apoptosis via the phosphorylation and activation of nSMase1 by JNK signaling. Cell Death Differ 22: 258-273

Yabu T, Shimuzu A \& Yamashita M (2009) A novel mitochondrial sphingomyelinase in zebrafish cells. J Biol Chem 284: 20349-20363

Zumbansen M \& Stoffel W (2002) Neutral sphingomyelinase 1 deficiency in the mouse causes no lipid storage disease. Mol Cell Biol 22: 3633-3638

\section{Figure legends}

\section{Figure 1: SMPD2 KD downregulates LAMP1 at the mRNA level}

(A) HeLa cells were transfected with the indicated siRNAs for $48 \mathrm{~h}$ and lysates were immunoblotted against LAMP1 and loading control GAPDH. (B) HeLa cells transfected with control (Ctrl) and SMPD2 siRNA for $48 \mathrm{~h}$ were stained with Lysotracker and DAPI and analyzed by confocal microscopy. (C) Quantifications of Lysotracker puncta per cell from (B). Unpaired Student's two-tailed t-test. (D) HeLa cells transfected with the indicated siRNAs for $48 \mathrm{~h}$ were then grown in FBS containing medium or serum-starved (starv) with or without Bafilomycin A (Baf) for 16 h. (E) Quantifications of lysosomal 
turnover of P62 and LC3B-II under basal conditions from (D). Ordinary one-way ANOVA followed by Dunnett's multiple comparisons test, not significant. (F) Quantifications of lysosomal turnover of P62 and LC3B-II under serum starvation conditions from (D). Ordinary one-way ANOVA followed by Dunnett's multiple comparisons test. (G) HeLa cells transfected with the indicated siRNA for $48 \mathrm{~h}$ were then treated with DMSO or MG132 for $16 \mathrm{~h}$. Lysates were immunoblotted against LAMP1, BiP, $\beta$-Catenin, and loading control GAPDH. (H) Real-time qPCR analysis of SMPD2 mRNA in HeLa cells after $48 \mathrm{~h}$ of SMPD2 KD. Unpaired Student's two-tailed $t$-test, ${ }^{* * * *} p<0.0001$. (I) Realtime qPCR analysis of LAMP1 mRNA in HeLa cells after $48 \mathrm{~h}$ of SMPD2 KD. Unpaired Student's two-tailed $t$-test, ${ }^{* * * *} \mathrm{p}<0.0001$. All the experiments from $(\mathrm{A})-(\mathrm{I})$ were done in at least three biological replicates.

\section{Figure 2: SMPD2 KD causes inefficient activation of ER stress signaling}

(A) HeLa cells treated with tunicamycin (Tuni) and Thapsigargin for $4 \mathrm{~h}$ and cell lysates were immunoblotted against nSMase1 and loading control GAPDH. (B) Western blot quantifications of nSMase1 from (A) normalized to the corresponding loading control GAPDH before normalization to the control. Ordinary one-way ANOVA followed by Dunnett's multiple comparisons test, ${ }^{*} p<0.01$ (C) HeLa cells transfected with Ctrl or SMP2 siRNA for $48 \mathrm{~h}$ were treated with tunicamycin or Thapsigargin for $4 \mathrm{~h}$. Cell lysates were immunoblotted against BiP, Calnexin, PDI, and loading control GAPDH. (D-E) Western blot quantifications of BiP (D), Calnexin (E), and PDI (F) normalized to the corresponding loading control GAPDH before normalization to the control. Ordinary oneway Anova followed by Tukey's multiple comparisons test. (G-M) Real-time qPCR analysis of spliced XBP-1 (sXBP-1) (G), ATF4 (H), EDEM (I), BiP (J), CHOP (K), LAMP1 (L), and SMPD2 (M) mRNA in HeLa cells transfected with Ctrl and SMPD2 siRNA for 48 $\mathrm{h}$ and treated with tunicamycin or thapsigargin for $4 \mathrm{~h}$. Ordinary one-way Anova followed by Tukey's multiple comparisons test, ${ }^{* * *} p<0.0001,{ }^{* *} p<0.0009{ }^{* *} p<0.001,{ }^{*} p<0.01$. (N) Viability assay of HeLa cells transfected with Ctrl, SMPD2, and UBC siRNA for $48 \mathrm{~h}$ and then treated with $4 \mu 8 \mathrm{C}(16 \mathrm{~h})$ or tunicamycin or thapsigargin for $4 \mathrm{~h}$. Ordinary oneway Anova followed by Tukey's multiple comparisons test, ${ }^{* * * *} p<0.0001,{ }^{* * *} p<0.0009$ ${ }^{* *} p<0.001,{ }^{*} p<0.01$. siRNA against UBC was used as a negative control for the viability 
assay. All experiments above, unless otherwise stated, are done in three biological replicates.

\section{Figure 3: SMPD2 KD arrests cells in the G1 phase}

(A) Percentage of HeLa cells transfected with Ctrl or SMPD2 siRNA for $48 \mathrm{~h}$ were analyzed by flow cytometry and sorted into Viable (Annexin- $\mathrm{V}^{-} / \mathrm{PI}^{-}$), Early apoptotic $\left(\right.$ Annexin- $\left.\mathrm{V}^{+} / \mathrm{Pl}^{-}\right)$, Late apoptotic $\left(\right.$Annexin $\left.-\mathrm{V}^{+} / \mathrm{PI}^{+}\right)$, and Necrotic (Annexin- $\left.\mathrm{V}^{-} / \mathrm{PI}^{+}\right)$. Twoway ANOVA followed by Sidak's multiple comparisons test, ${ }^{* *} p<0.001,{ }^{*} p<0.01$. (B) HeLa cells were transfected with Ctrl or SMPD2 siRNA for $48 \mathrm{~h}$ and then cell lysates were immunoblotted against different apoptosis markers and loading control GAPDH. (C) HeLa cells transfected with Ctrl or SMPD siRNA for $48 \mathrm{~h}$ were replated and seeded into a 96well plate for Incucyte proliferation analysis. Proliferation rate was measured by cell confluence percent versus time by taking images of the wells at $2 \mathrm{~h}$ intervals for the indicated duration. Paired Student's two-tailed $t$-test, ${ }^{* * *} \mathrm{p}<0.0001$. (D) HeLa cell mean diameter (Left) and peak diameter (Right) determined with Casy cell counter after $72 \mathrm{~h}$ of Ctrl or SMPD2 siRNA transfection. Unpaired Student's two-tailed $t$-test, ${ }^{*} p<0.01$. (E-F) Percentage of HeLa cells transfected with Ctrl or SMPD2 siRNA for $48 \mathrm{~h}(\mathbf{E})$ and $72 \mathrm{~h}(\mathbf{F})$ were gated for $\mathrm{G} 1, \mathrm{~S}$, and $\mathrm{G} 2 / \mathrm{M}$. Mitotic index in $(\mathrm{E})$ was determined from the G2/M population positive for p-Mpm-2 staining. Two-way ANOVA followed by Sidak's multiple comparisons test, ${ }^{* * *} p<0.0001,{ }^{* * *} p<0.0009{ }^{* *} p<0.001$. (G) Lysates from HeLa cells transfected with Ctrl or SMPD2 siRNA for $72 \mathrm{~h}$ were immunoblotted against P27, P53, P21, and loading control GAPDH. (H) Western blot quantifications of P21 (Left) and P27 (Right) normalized to the corresponding loading control GAPDH before normalization to the control. Unpaired Student's two-tailed t-test, ${ }^{\star * * *} p<0.0001,{ }^{* *} p<0.001$. All experiments from $(A)$ to $(H)$ are done in three biological replicates.

\section{Figure 4: Wnt signaling is downregulated in SMPD2 KD cells}

(A) Scheme showing P53-dependent DNA damage response pathway that mediates G1 cell cycle arrest. (B) Lysates from HeLa cells transfected with Ctrl or SMPD2 siRNA for $48 \mathrm{~h}$ were immunoblotted against the $\mathrm{p}$-Chk2, p-Chk1, p-Rb, and loading control GAPDH.

(C) Western blot quantifications of $\mathrm{p}$-Chk1 and p-Ch2 from (B) normalized to the corresponding loading control GAPDH before normalization to the control. Unpaired Student's two-tailed $t$-test, ${ }^{* *} p<0.001$. (D) Scheme showing PI3K/Akt signaling pathway. 
(E) Lysates from HeLa cells transfected with Ctrl or SMPD2 siRNA for $48 \mathrm{~h}$ were immunoblotted against the p-Akt, p-PTEN, and loading control GAPDH. (F) Western blot quantifications of $\mathrm{p}$-Akt and p-PTEN from (E) normalized to the corresponding loading control GAPDH before normalization to the control. Unpaired Student's two-tailed $t$-test, ${ }^{* *} \mathrm{p}<0.001$. (G) Wnt reporter activity analysis in HCT116 cells transfected with the indicated siRNAs for $72 \mathrm{~h}$ measured using TCF/LEF luciferase reporter assay. The reporter vectors were transected $24 \mathrm{~h}$ after siRNA reverse transfection. Ordinary one-way Anova followed by Tukey's multiple comparisons test, ${ }^{* * *} p<0.0001,{ }^{*} p<0.01$. (H) HCT116 cells were transfected with Ctrl or SMPD2 siRNAs for $72 \mathrm{~h}$ and lysates were immunoblotted against $\beta$-catenin, Wnt3a, and loading control GAPDH. (I) Western blot quantifications from (I) normalized to the corresponding loading control GAPDH before normalization to the control. Unpaired Student's two-tailed $t$-test, ${ }^{* *} p<0.0009$. (H-L) (J) Real-time qPCR analysis of SMPD2, WNT3A, and AXIN2 mRNA in HCT116 cells transfected with Ctrl and SMPD2 siRNA for $72 \mathrm{~h}$. Unpaired Student's two-tailed t-test, ${ }^{* * * *} \mathrm{p}<0.0001$. (K) HeLa cells were transfected with Ctrl or SMPD2 siRNA for $48 \mathrm{~h}$ and treated with or without Cycloheximide $(\mathrm{CHX})$ for $16 \mathrm{~h}$. Lysates, after labeling with puromycin for $1 \mathrm{~h}$, were immunoblotted against puromycinylated proteins with puromycin antibody. (L) Western blot quantifications from (K) normalized to the corresponding loading control GAPDH before normalization to the control. Ordinary one-way Anova followed by Tukey's multiple comparisons test, ${ }^{* * *} p<0.0001,{ }^{* * *} p<0.009$. All experiments from $(A)-(L)$ are done in three biological replicates.

\section{Supplementary Figure 1:}

(A) Viability assay of HeLa cells transfected with Ctrl, SDCBP, NSMAF, SMPD2, and SMPD3 siRNA for $48 \mathrm{~h}$. Ordinary one-way ANOVA followed by Dunnett's multiple comparisons test, ${ }^{* * \star} p<0.0001$. (B) Western blot quantifications of LAMP1 from (Fig.1A) normalized to the corresponding loading control GAPDH before normalization to the control. Ordinary one-way ANOVA followed by Dunnett's multiple comparisons test, ${ }^{* * *} p$ $<0.0009$. (C) HCT116 cells were transfected with the indicated siRNAs for $48 \mathrm{~h}$ and lysates were immunoblotted against LAMP1 and loading control GAPDH. (D) Western blot quantifications of LAMP1 from (Fig.1G) normalized to the corresponding loading control GAPDH before normalization to the control. Ordinary one-way ANOVA followed 
by Dunnett's multiple comparisons test, ${ }^{*} p<0.01$. (E-F) Real-time qPCR analysis of LAMP1 (E) and LAMP2 (F) mRNA in HCT116 cells after $72 \mathrm{~h}$ of SMPD2 KD. Unpaired Student's two-tailed $t$-test, ${ }^{* * * *} \mathrm{p}<0.0001$. (G) HeLa cells transfected with control (Ctrl) and SMPD2 siRNA for $48 \mathrm{~h}$ were stained for ceramide and calnexin and analyzed by confocal microscopy. (H) Quantifications of mean intensity of ceramide puncta per cell from (G) (left) and mean intensity of ceramide puncta colocalizing with calnexin (right) from three independent experiments. Unpaired Student's two-tailed $t$-test, ${ }^{* \star * *} \mathrm{p}<0.0001$. (I) HeLa cells transfected with Ctrl or SMPD2 siRNA for $48 \mathrm{~h}$ were live imaged by staining with ER tracker and DAPI. (J) Real-time qPCR analysis of sXBP-1 mRNA in HeLa cells transfected with Ctrl and SMPD2 siRNA and treated with $4 \mu 8 \mathrm{C}$ for $48 \mathrm{~h}$. Ordinary oneway ANOVA followed by Dunnett's multiple comparisons test, ${ }^{* * *} p<0.0001,{ }^{*} p<0.01$, from two independent experiments. (K) Real-time qPCR analysis of LAMP1 mRNA in HeLa cells transfected with Ctrl or SMPD2 siRNA and treated with $4 \mu 8 \mathrm{C}$ for $48 \mathrm{~h}$. Ordinary one-way ANOVA followed by Dunnett's multiple comparisons test, ${ }^{* * *} p<0.0001,{ }^{* *} p<$ 0.001, " $p<0.01$, from two independent experiments. (L) HeLa cells reverse transfected with Ctrl and SMPD2 siRNA were grown with DMSO or $4 \mu 8 \mathrm{C}$ for $48 \mathrm{~h}$ and treated with tunicamycin for $4 \mathrm{~h}$. Cell lysates were immunoblotted against nSMase1, LAMP1, and loading control GAPDH. All experiments from (A)-(L) are done in three biological replicates.

\section{Supplementary Figure 2:}

(A) Flow Cytometry analysis of FITC Annexin-V and PI staining of HeLa cells transfected with Ctrl (left) or SMPD2 (right) siRNA for $48 \mathrm{~h}$. Forward and side scatter plot (above left) of HeLa cells gated for the subsequent analysis, two-dimensional scatter plot gating cell populations into four quadrants based on PI versus FITC Annexin-V staining (above right), Populations of cells gated for only FITC Annexin-V (below left) and only PI (below right). (B-E) Western blot quantifications of cleaved caspase 3 (B), caspase 7 (C), PARP I (D), and PARP I| (E) in relative to the respective full length (FL) proteins from two independent experiments. (F) HCT116 cell mean diameter (above) and peak diameter (bottom) determined with Casy cell counter after $72 \mathrm{~h}$ of Ctrl or SMPD2 siRNA transfection. (G) Flow cytometry analysis HeLa cells transfected with Ctrl (left) or SMPD2 (right) siRNA for $48 \mathrm{~h}$ were gated to distribute the cell population into $\mathrm{G} 1, \mathrm{~S}$, and G2/M 
phase of the cell cycle $(\mathbf{H})$ Percentage of HeLa cells transfected with Ctrl or SMPD2 siRNA for $96 \mathrm{~h}$ were gated for G1, S, and G2/M. Two-way ANOVA followed by Sidak's multiple comparisons test, ${ }^{* * *} p<0.0001,{ }^{* *} p<0.0009{ }^{* *} p<0.001$. All experiments from (A)-(I) are done in three biological replicates unless otherwise stated.

\section{Supplementary Figure 3:}

(A) Western blot quantifications of P53 protein levels from (Fig. 3G) normalized to the corresponding loading control GAPDH before normalization to the control. Unpaired Student's two-tailed t-test). (B) Lysates from HeLa cells transfected with Ctrl or SMPD2 siRNA for $96 \mathrm{~h}$ were immunoblotted against P27, P53, P21, and loading control GAPDH. (C) Western blot quantifications from (B) of P27, P21, and P53 normalized to the corresponding loading control GAPDH before normalization to the control. Unpaired Student's two-tailed t-test, ${ }^{* * *} p<0.0001,{ }^{* *} p<0.001$ (D) Western blot quantifications of $\mathrm{p}-\mathrm{Rb}$ from (Fig. 4B) normalized to the corresponding loading control GAPDH before normalization to the control. Unpaired Student's two-tailed t-test, ${ }^{* *} p<0.001$. (E) HeLa cells were transfected with the indicated siRNAs for $48 \mathrm{~h}$ and lysates were immunoblotted against $\beta$-catenin and loading control GAPDH. (F) Western blot quantifications of $\beta$ catenin from (E) normalized to the corresponding loading control GAPDH before normalization to the control. Ordinary one-way ANOVA followed by Dunnett's multiple comparisons test, ${ }^{*} p<0.01$. (G) Real-time qPCR analysis of CTNNB-1, EVI, WNT7B, and GAPDH mRNA in HCT116 cells transfected with Ctrl and SMPD2 siRNA for $72 \mathrm{~h}$. Unpaired Student's two-tailed t-test. $(\mathbf{H})$ Real-time qPCR analysis of SMPD4 mRNA in HCT116 cells transfected with Ctrl and SMPD2 siRNA for $72 \mathrm{~h}$. Unpaired Student's twotailed $t$-test, ${ }^{* \star \star *} p<0.0001$. All experiments from $(\mathrm{A})-(\mathrm{H})$ are done in three biological replicates. 

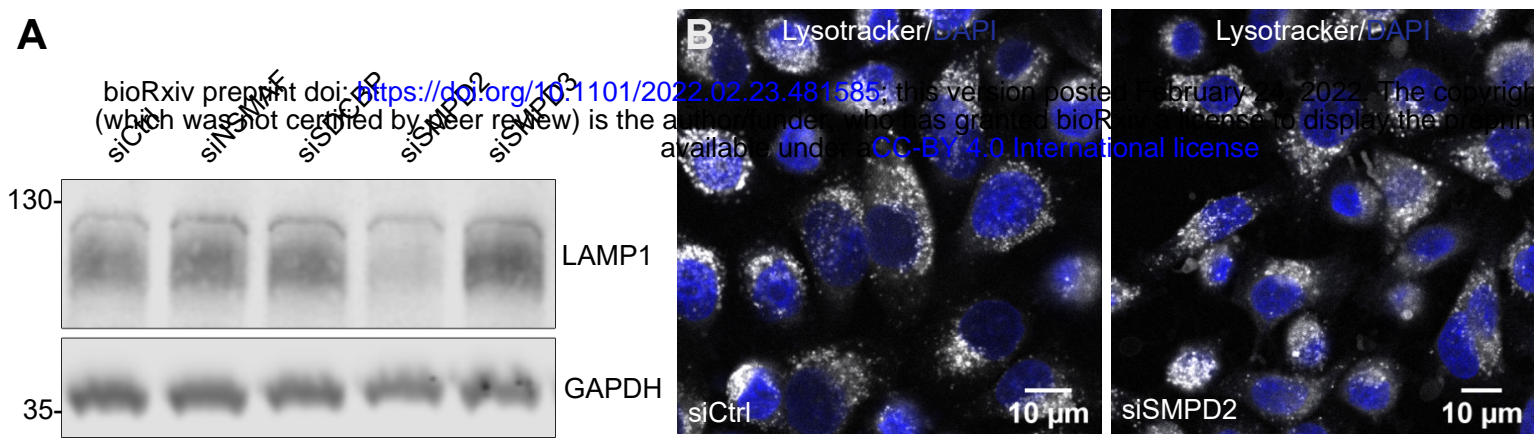

\section{C}

t holdgogfor this preprint inforpety ity. It is made 放 $600-$ ns

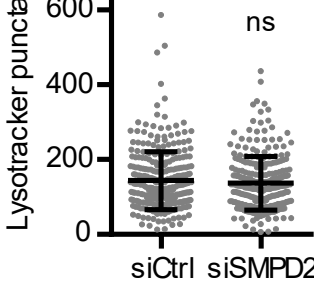

D

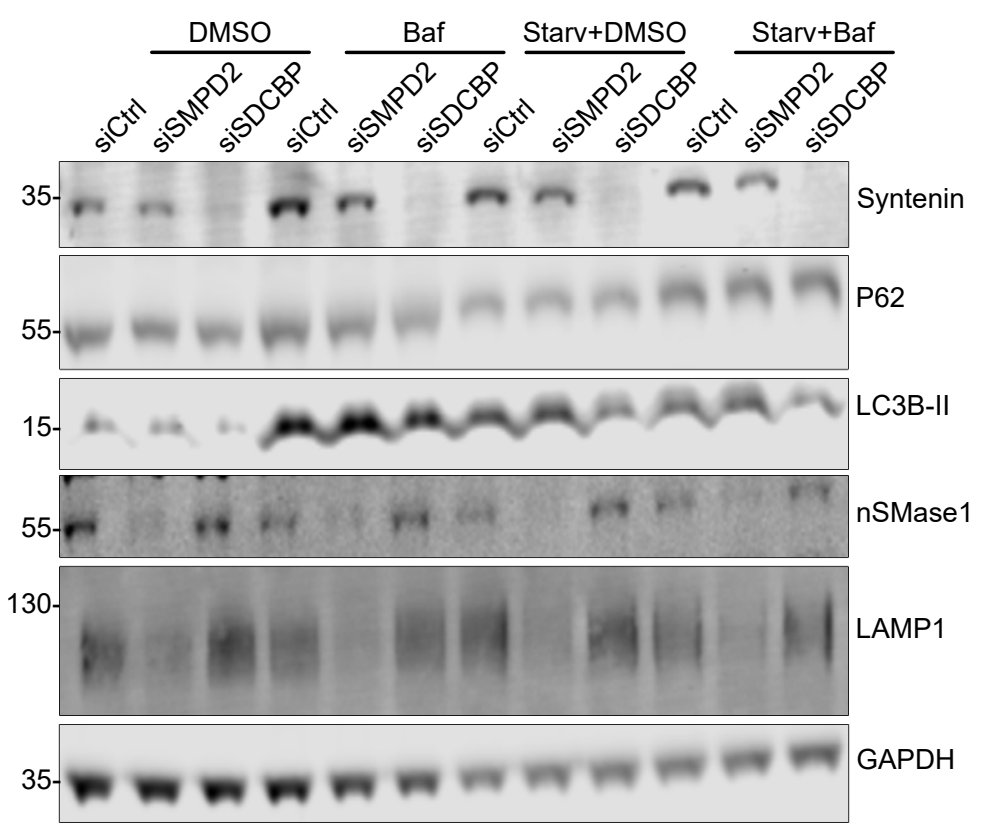

G
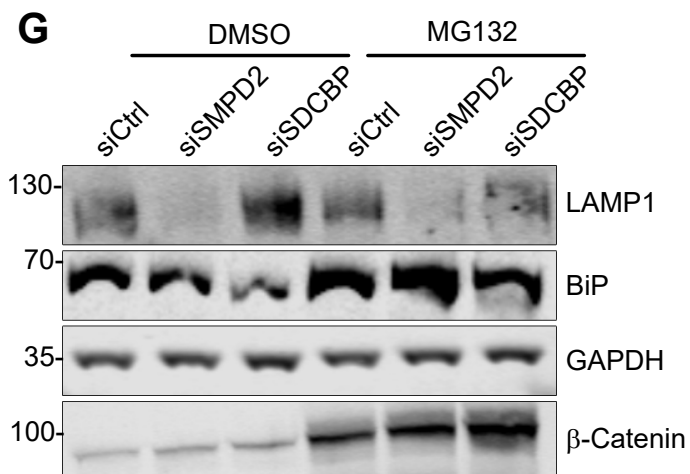

E
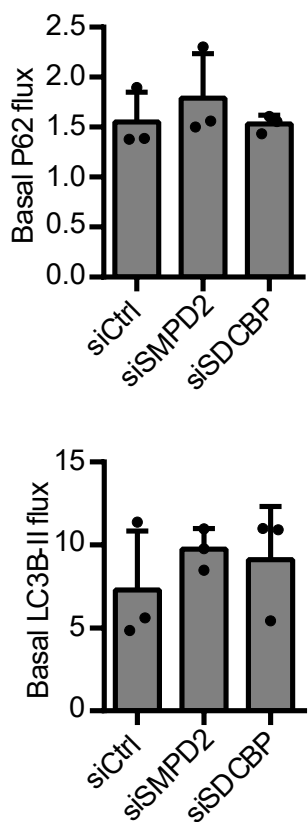

I

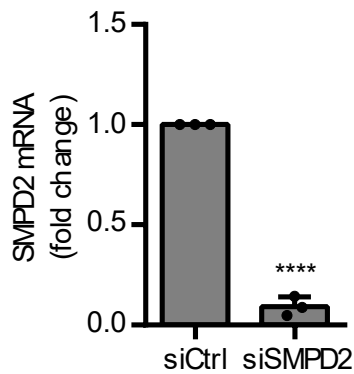

$\mathbf{F}$
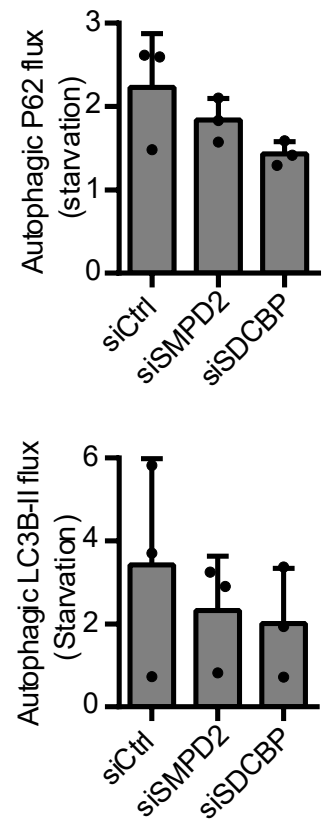

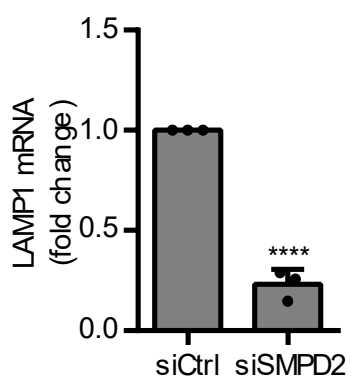


A

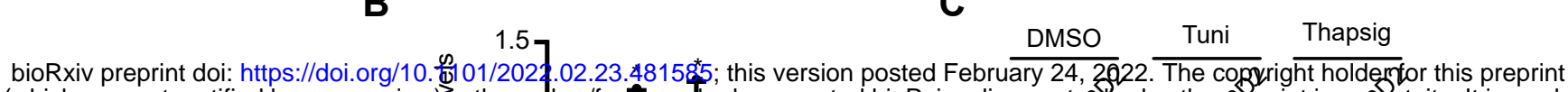
(which was not certified by peer reviewe DMSO Tuni Thapsig
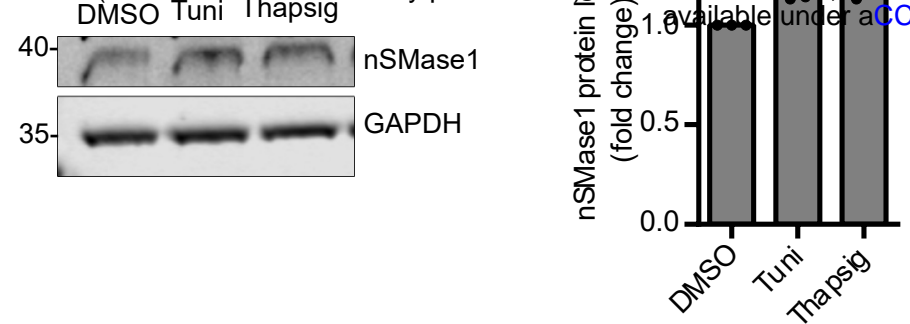

$\mathbf{E}$
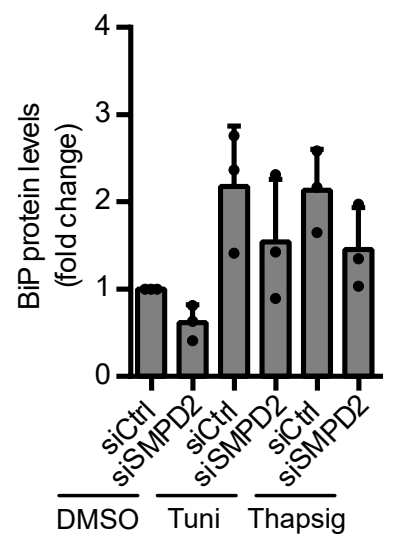

G

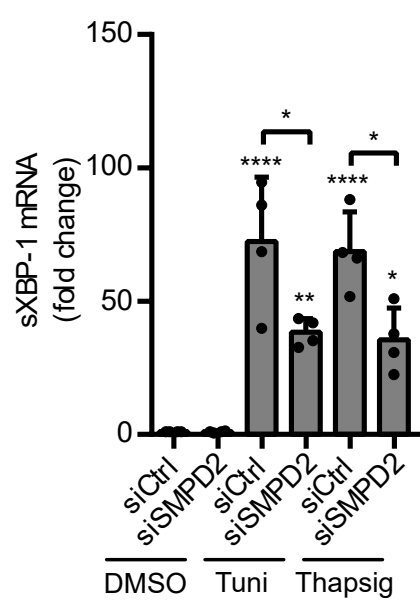

K
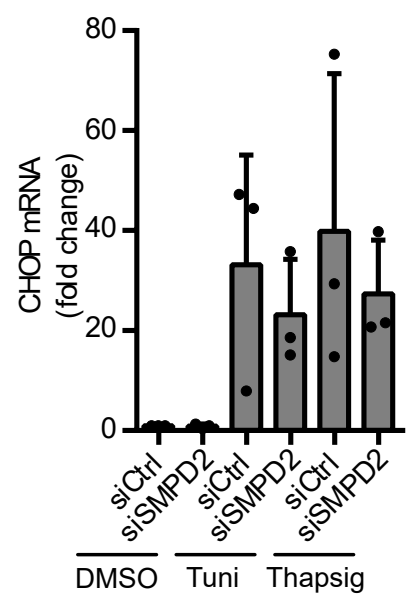

$\mathbf{H}$

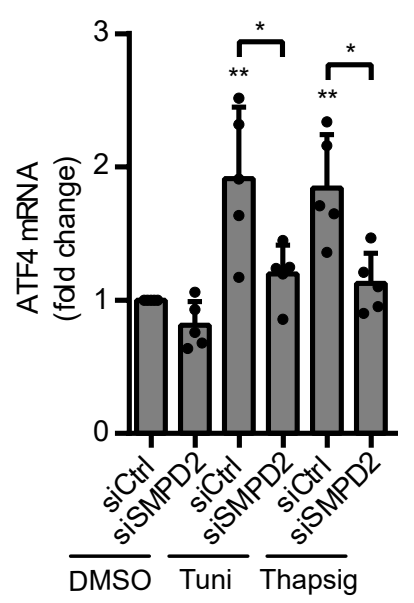

$\mathbf{L}$

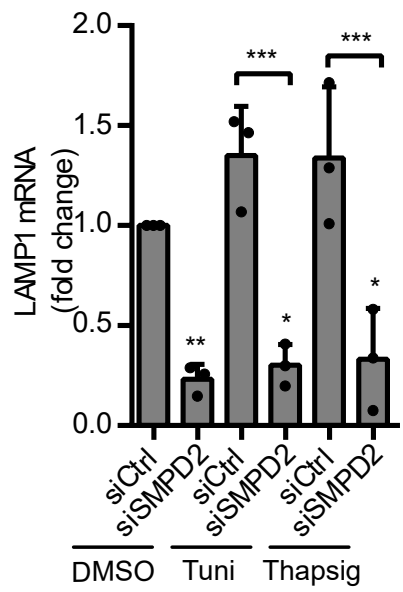

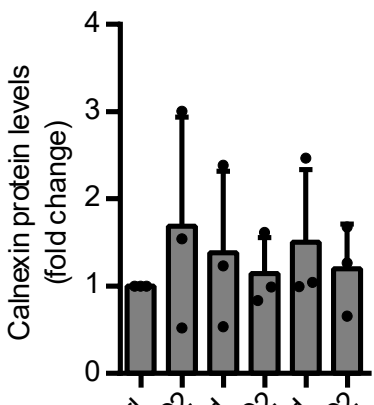

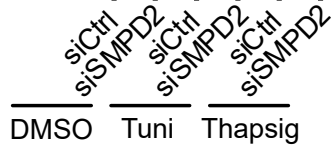

I

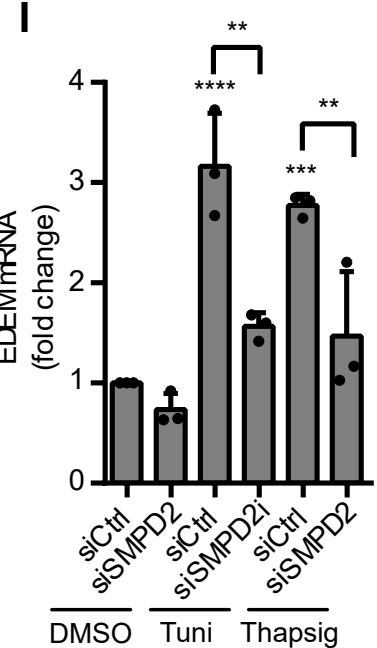

M

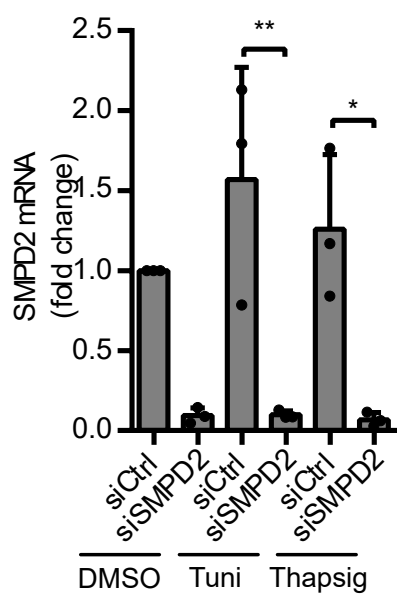

$\mathbf{F}$

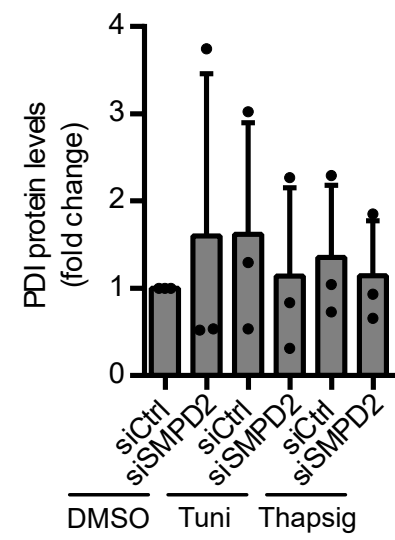

$J$

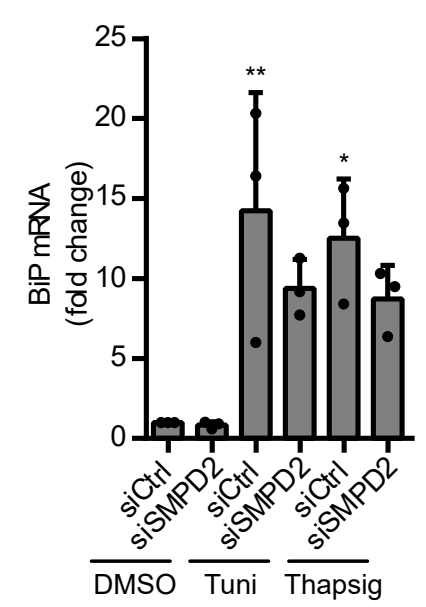

$\mathbf{N}$

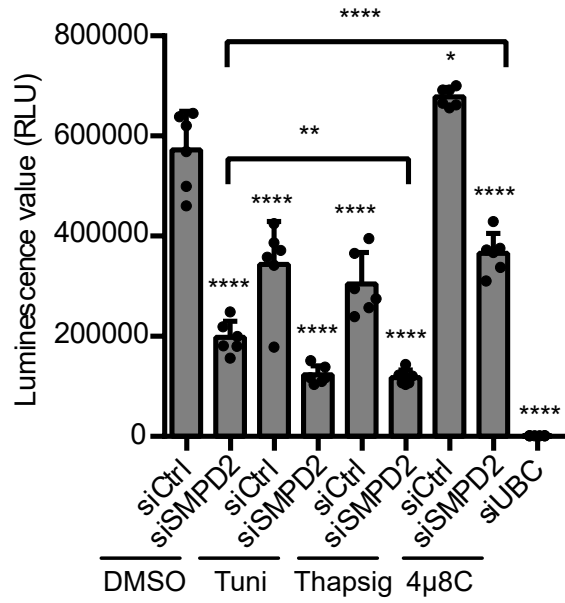


bioRxiv preprint doi: https://doi.org/10.1101/2022.023.23.49-585; thinersion posted Eebruary 24, 2022. The copyright holder for this preprint (which was not certified by peer review) is the author/funaer, who has granted biokxiv a license to display the preprint in perpetuity. It is made

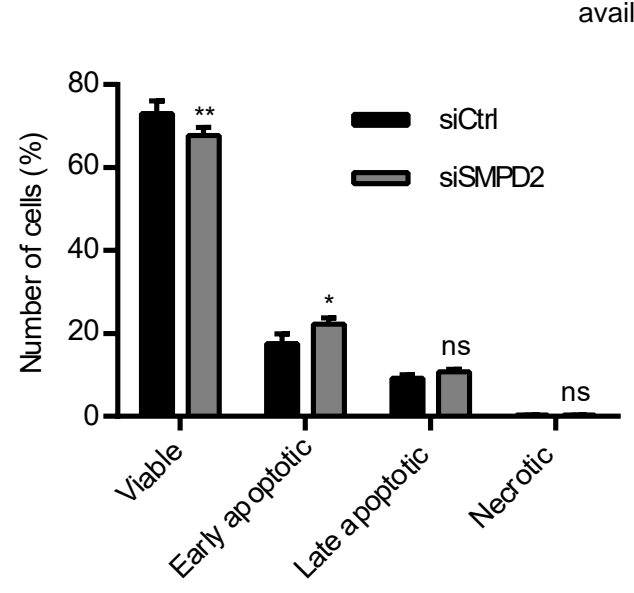
available under aCC-BY 4.0 International license.

D

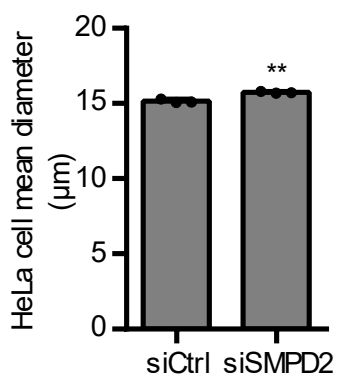

G

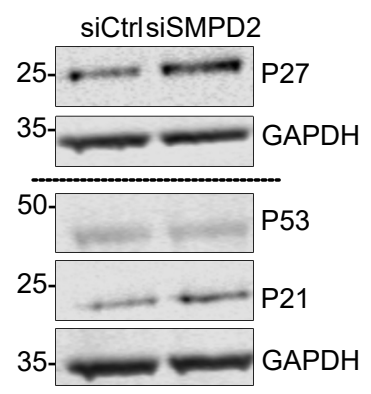

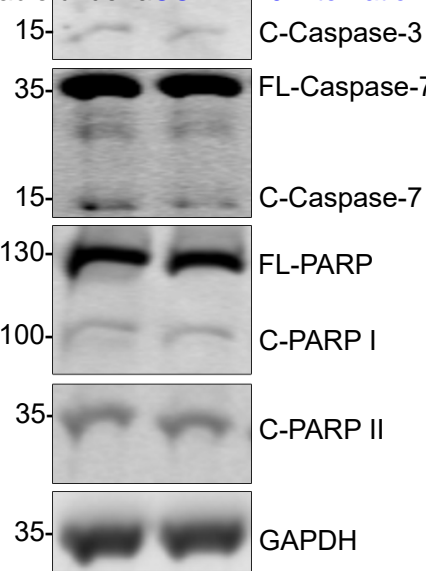

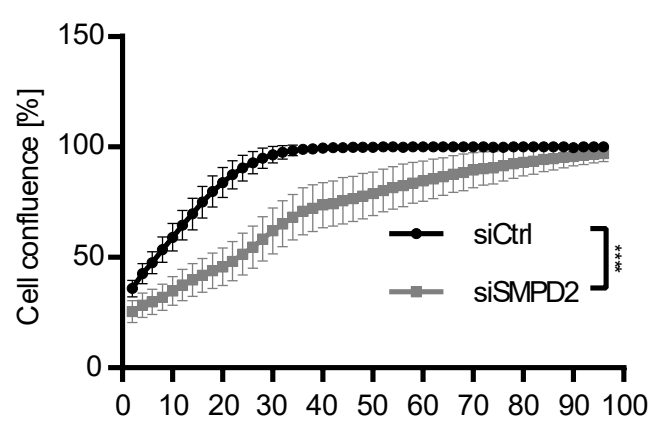

E
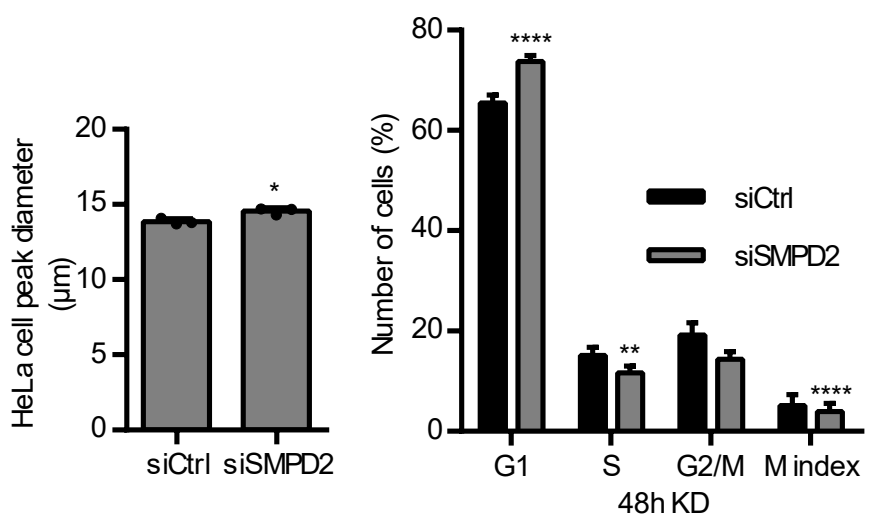

H
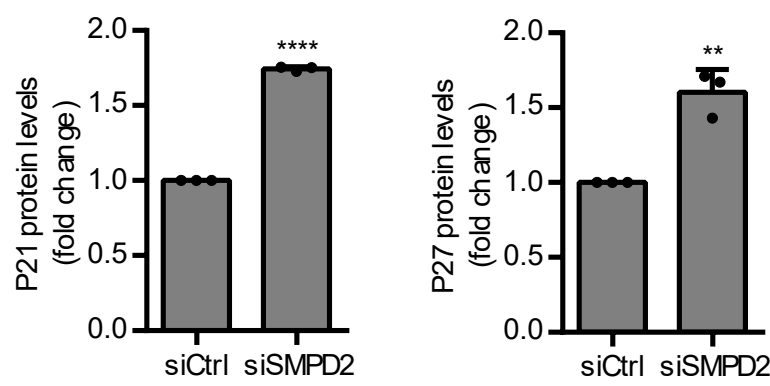
A

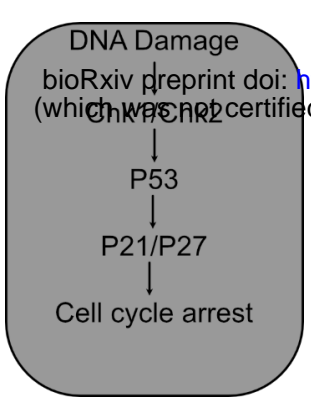

D

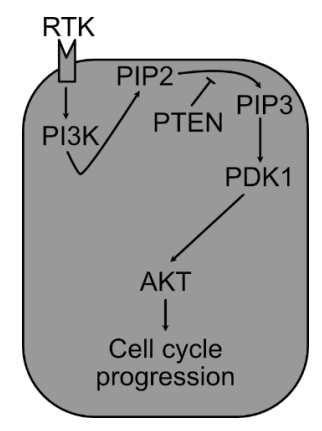

G
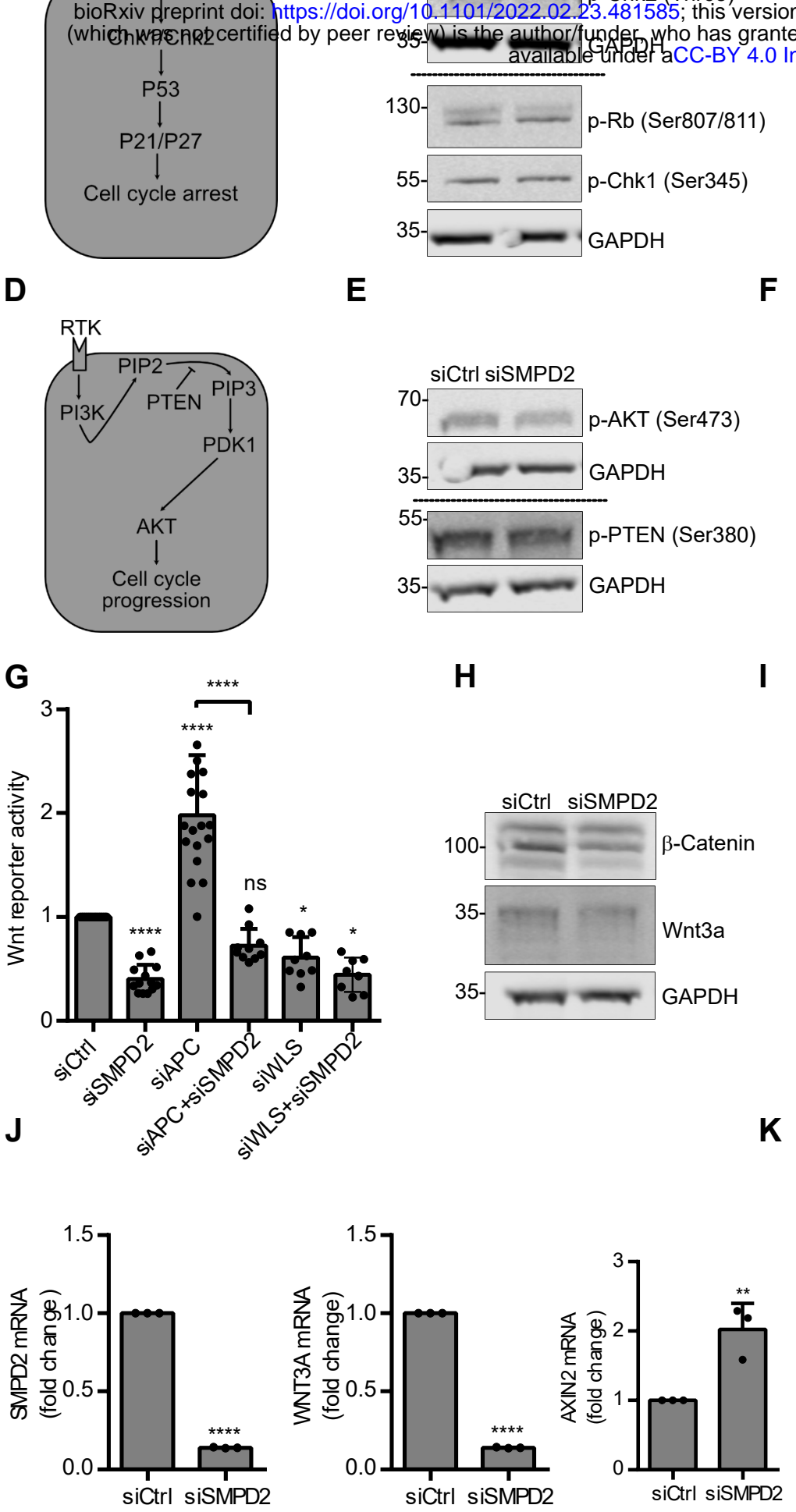

E

F

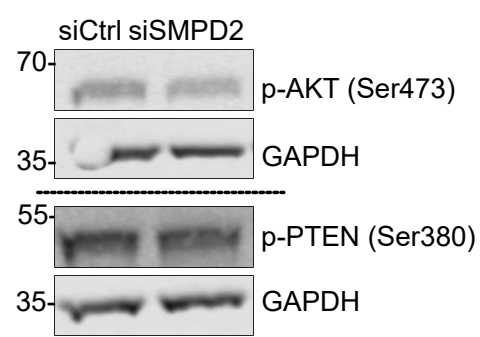

H

I

K
C

$\frac{\infty}{0} \quad 1.5$

$\frac{\infty}{\Phi} \quad 1.5$

for this preprint @osted Fepruary 24, 2022. The copyyght holde tor his preprint
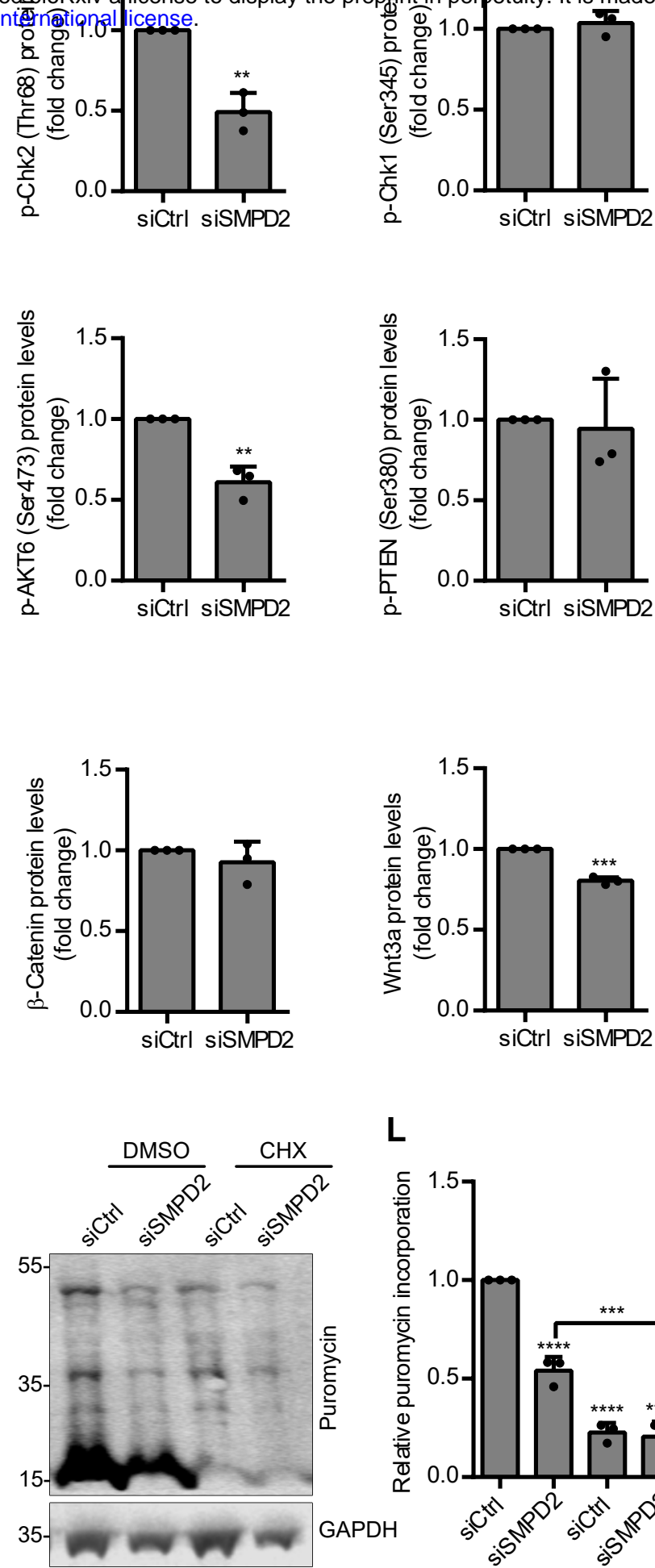

L

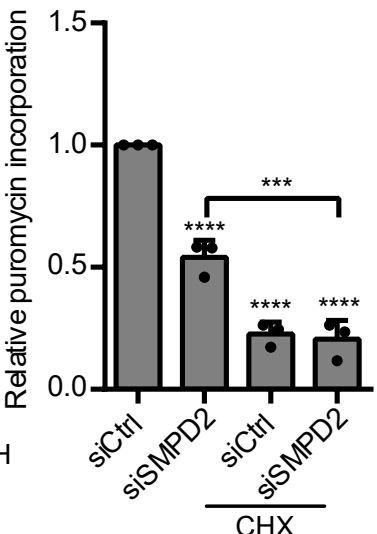




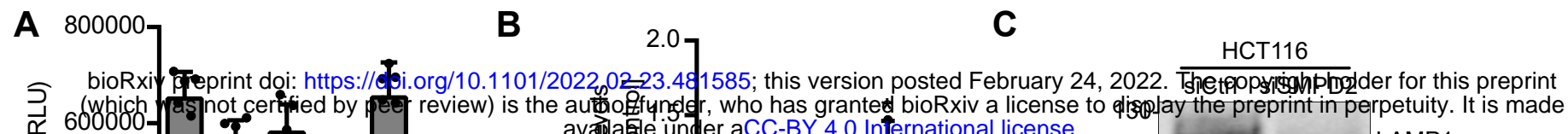

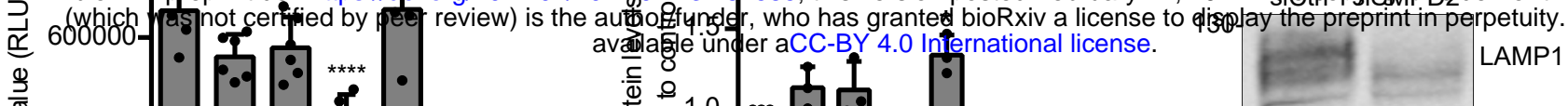

400000-

200000

D
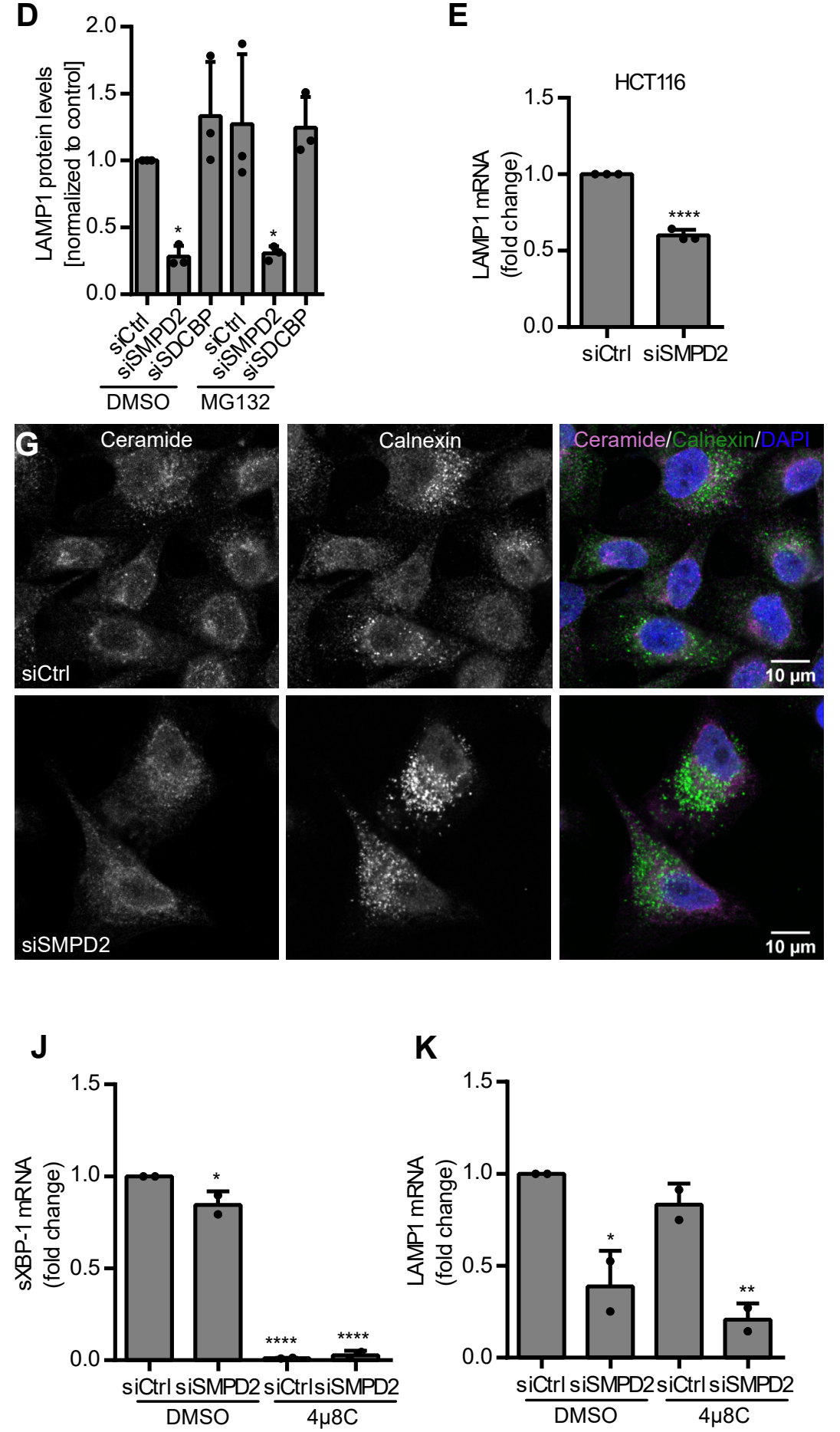

$\mathbf{F}$
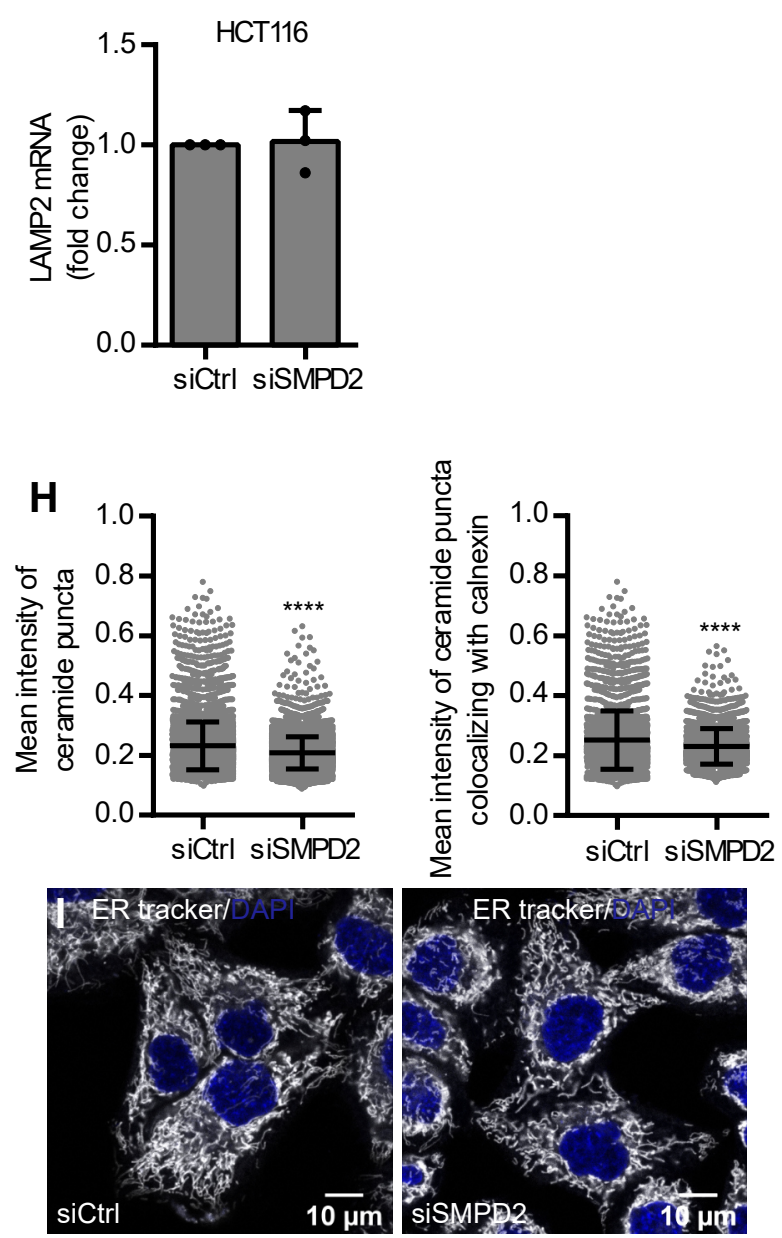

L

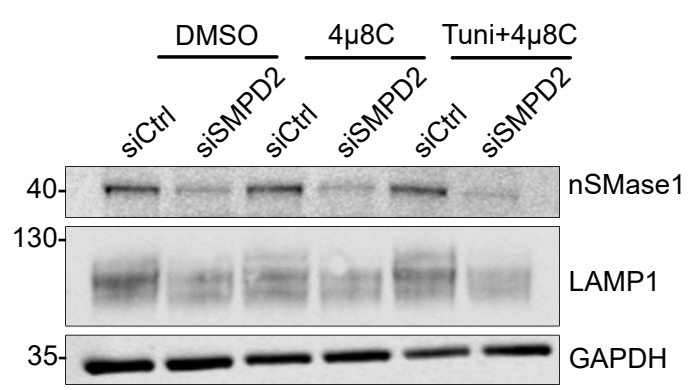


A

siCtrl

SiSMPD2

bioRxiv preprint doi: https://doi.org/10.1101/2022.02.23.481585; this version posted February 24, 2022. The copyright holder for this preprint

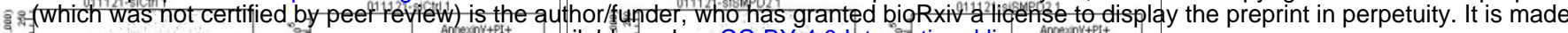
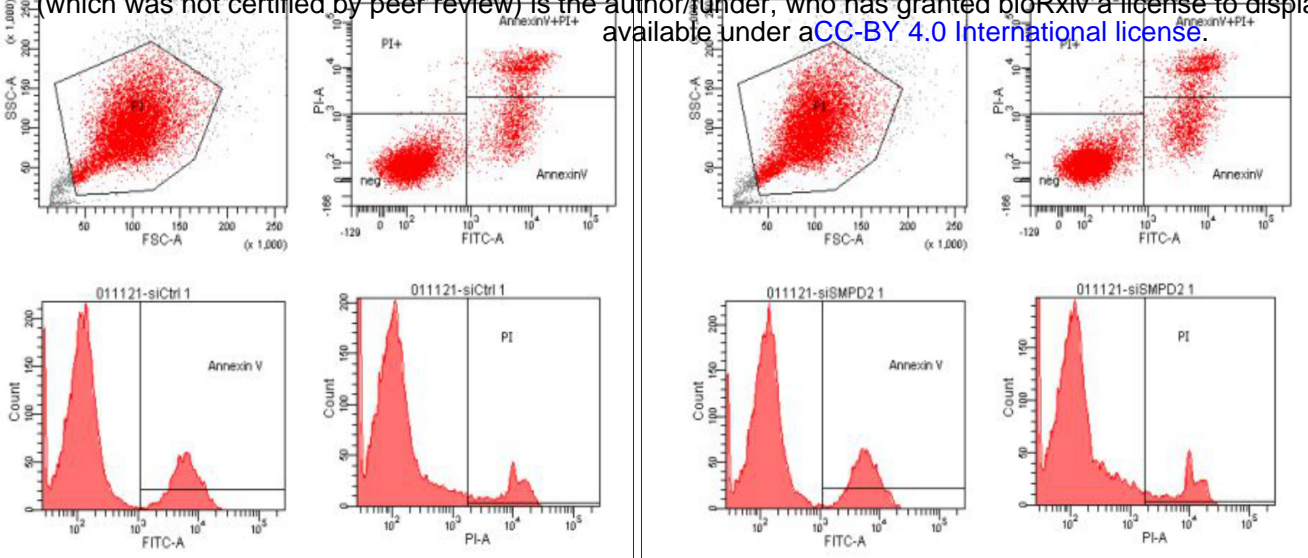

B
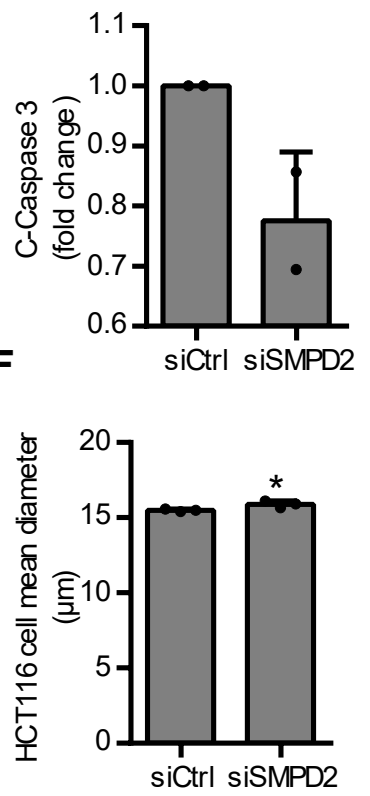

G

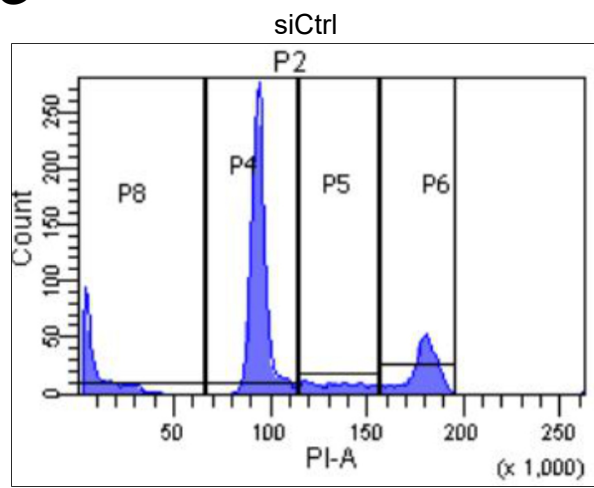

C
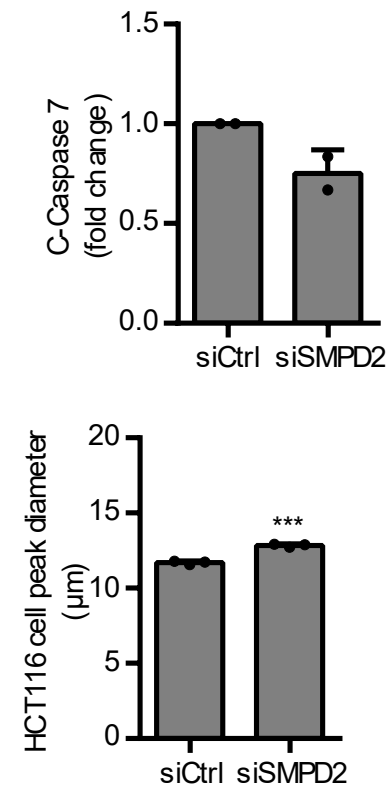

D

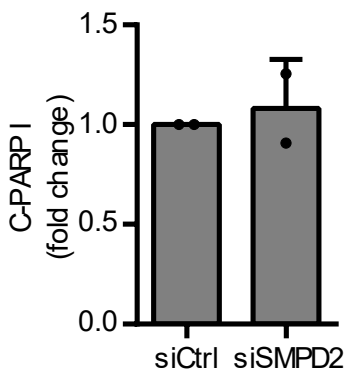

E

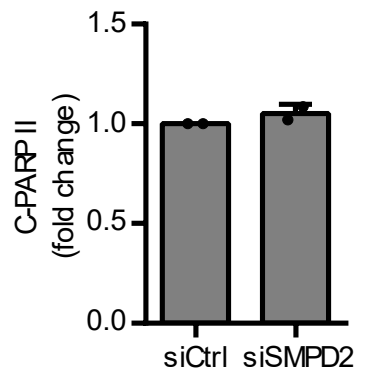

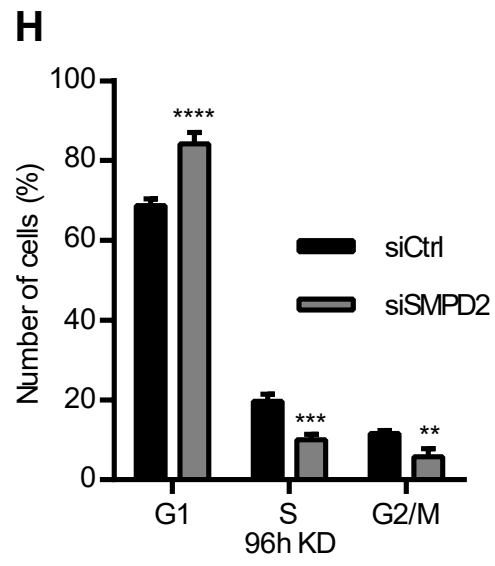


A

B $\frac{96 \mathrm{~h}}{\text { siCtrl siSMPD2 }}$

C

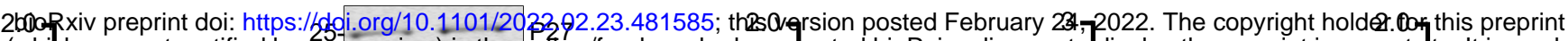
(whifh was not certified by peer review) is the author/funder, who has g anted bioRxiv a license to display the preprint in perpetulty. It is made

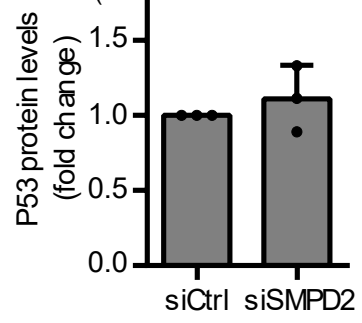

D

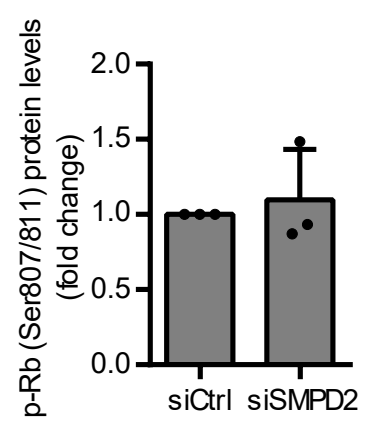

$\mathbf{E}$

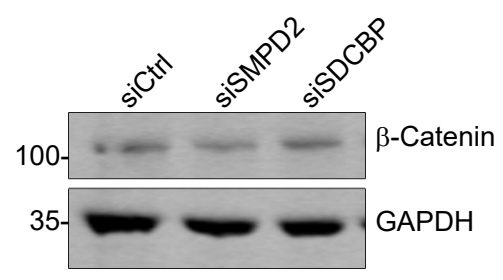

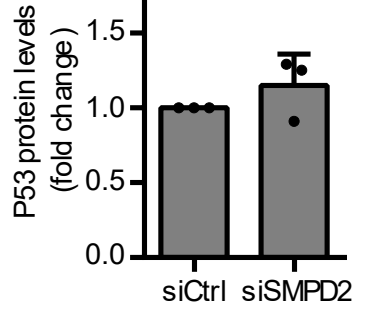

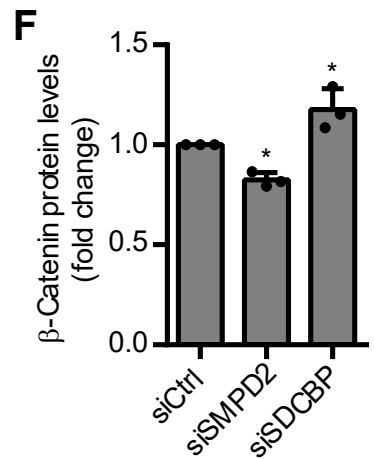

G

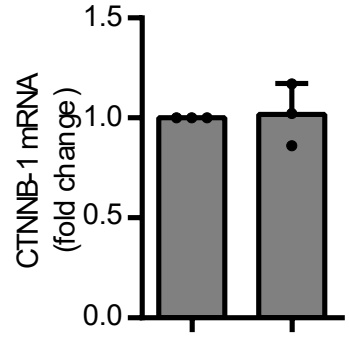

H

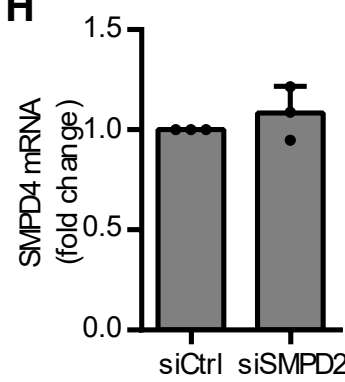

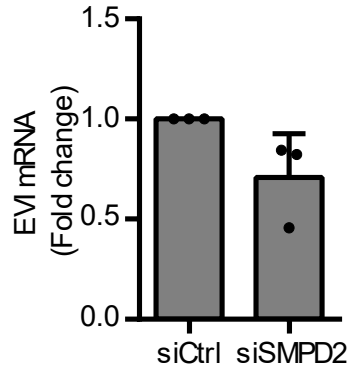

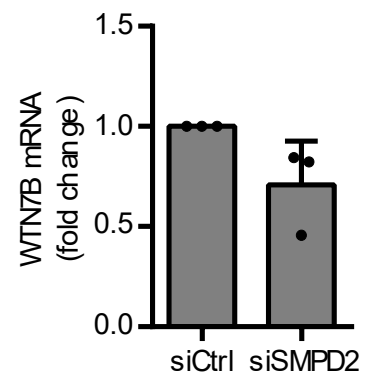

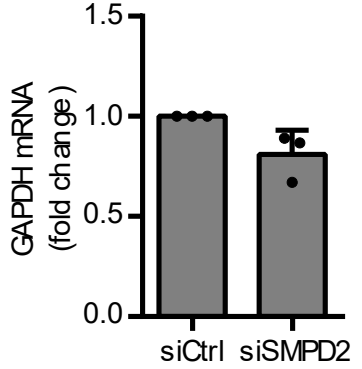

